ARTICLE

https://doi.org/10.1038/s41467-019-11872-9

\title{
An ancestral NB-LRR with duplicated 3'UTRs confers stripe rust resistance in wheat and barley
}

Chaozhong Zhang (10 1,2,9 Lin Huang ${ }^{3,9}$, Huifei Zhang ${ }^{2}$, Qunqun Hao ${ }^{2}$, Bo Lyu², Meinan Wang ${ }^{4}$, Lynn Epstein (1) ${ }^{5}$,

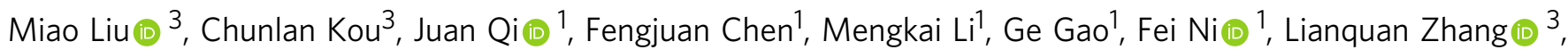
Ming $\mathrm{Hao}^{3}$, Jirui Wang ${ }^{3}$, Xianming Chen (1) 6, Ming-Cheng Luo (1) ${ }^{7}$, Youliang Zheng ${ }^{3}$, Jiajie Wu (D) ${ }^{1}$, Dengcai Liu (i) ${ }^{3,8} \&$ Daolin Fu (1) ${ }^{2}$

Wheat stripe rust, caused by Puccinia striiformis f. sp. tritici (Pst), is a global threat to wheat production. Aegilops tauschii, one of the wheat progenitors, carries the YrAS2388 locus for resistance to Pst on chromosome 4DS. We reveal that YrAS2388 encodes a typical nucleotide oligomerization domain-like receptor (NLR). The Pst-resistant allele YrAS2388R has duplicated $3^{\prime}$ untranslated regions and is characterized by alternative splicing in the nucleotidebinding domain. Mutation of the YrAS2388R allele disrupts its resistance to Pst in synthetic hexaploid wheat; transgenic plants with YrAS2388R show resistance to eleven Pst races in common wheat and one race of $P$. striiformis f. sp. hordei in barley. The YrAS2388R allele occurs only in Ae. tauschii and the Ae. tauschii-derived synthetic wheat; it is absent in $100 \%$ $(n=461)$ of common wheat lines tested. The cloning of YrAS2388R will facilitate breeding for stripe rust resistance in wheat and other Triticeae species.

\footnotetext{
${ }^{1}$ State Key Laboratory of Crop Biology, Shandong Agricultural University, 271018 Tai'an, Shandong, China. ${ }^{2}$ Department of Plant Sciences, University of Idaho, Moscow, ID 83844, USA. ${ }^{3}$ Triticeae Research Institute, Sichuan Agricultural University, 611130 Chengdu, Sichuan, China. ${ }^{4}$ Department of Plant Pathology, Washington State University, Pullman, WA 99164, USA. ${ }^{5}$ Department of Plant Pathology, University of California, Davis, CA 95616, USA. ${ }^{6}$ Wheat Health, Genetics, and Quality Research Unit, USDA-ARS, Pullman, WA 99164, USA. ${ }^{7}$ Department of Plant Sciences, University of California, Davis, CA 95616, USA. ${ }^{8}$ State Key Laboratory of Crop Gene Exploration and Utilization in Southwest China, Sichuan Agricultural University, 611130 Chengdu, Sichuan, China. ${ }^{9}$ These authors contributed equally: Chaozhong Zhang, Lin Huang. Correspondence and requests for materials should be addressed to J.W. (email: jiajiewu@sdau.edu.cn) or to D.L. (email: dcliu7@yahoo.com) or to D.F. (email: dlfu@uidaho.edu)
} 
W heat (Triticum spp.) is the largest acreage crop in the world. With an approximate 220 million hectares and 760 million tons in 2018, wheat was ranked second in global production after maize ${ }^{1}$. As a staple food crop, wheat provides about $20 \%$ of global calories for human consumption ${ }^{2}$. Because the world population is projected to increase by nearly two billion people within the next three decades ${ }^{3}$, the increasing human population worldwide will place an even greater demand for wheat production globally.

Wheat stripe rust (or yellow rust; abbreviated as Yr), caused by Puccinia striiformis $\mathrm{f}$. sp. tritici (Pst), is a serious fungal disease that poses a huge threat to wheat production in regions with cool and moist weather conditions ${ }^{4}$, including major wheat-producing countries, such as Australia, Canada, China, France, India, the United States, and many others ${ }^{5,6}$. Planting wheat cultivars with adequate levels of resistance is the most practical and sustainable method to control stripe rust. Host resistance of wheat against Pst is normally classified as either all-stage resistance (ASR) or adultplant resistance (APR). Whereas ASR is effective starting at the seedling stage through the late stages of plant growth, APR is mainly effective at the late stages of plant growth ${ }^{7}$. In wheat, ASR confers high levels of resistance to specific Pst races, but the underlying genes, such as $Y r 9^{8}$ and $Y r 17^{9}$, are often circumvented by the emergence of new virulent races. In contrast, APR typically provides a partial level of resistance, but is more durable and is effective against all or a wider spectrum of Pst races than ASR. High-temperature adult-plant (HTAP) resistance is a major type of APR; HTAP typically provides durable and non-race-specific resistance to $P s t^{10}$. Incorporating multiple ASR and HTAP genes appears to be an excellent strategy for maintaining sustainable resistance to wheat stripe rust ${ }^{10}$.

Over 80 wheat stripe rust resistance $(R)$ genes $(Y r 1-Y r 81)$ have been permanently named ${ }^{11}$. Of the seven genes cloned so far, $Y r 5$, $Y r 7$ and $Y r S P$, a gene cluster, encodes nucleotide-binding (NB) and leucine-rich repeat (LRR) proteins ${ }^{12}$; Yr15 has two kinaselike domains ${ }^{13}$; Yr36 has a kinase domain and a lipid binding domain ${ }^{14}$; Lr34/Yr18 encodes a putative ABC transporter ${ }^{15}$; and Lr67/Yr46 encodes a predicted hexose transporter ${ }^{16}$. While the Yr5/Yr7/YrSP cluster and Yr15 confer ASR resistance to wheat stripe rust, $Y r 18, Y r 36$, and Yr46 confer APR or HTAP resistance. Of the three cloned APR genes, only the $\operatorname{Yr} 18$ gene has been widely used in wheat cultivars ${ }^{17}$; however, $\operatorname{Yr} 18$ alone does not confer adequate resistance under high disease pressures. $Y r 7$ and $Y r S P$ confer high levels of resistance, but Pst races virulent to $\mathrm{Yr} 7$ are common globally and those virulent to $Y r S P$ occur in some countries $^{18}$. Yr5 and $\operatorname{Yr} 15$ confer high levels of resistance to a wide range of Pst races ${ }^{12,19}$, but the increasing adoption of them in wheat cultivars may cause the emergence of virulent races. Characterization of additional $R$ genes is essential in order to assemble effective resistance to constantly changing populations of Pst.

Aegilops tauschii Coss. $(2 n=2 \times=14, \mathrm{DD})$ is the D genome progenitor of common wheat ${ }^{20,21}$. The diverse Ae. tauschii $\mathrm{D}$ genome offers a valuable gene pool for stripe rust resistance 20,22 . To date, several stripe rust resistance genes have been mapped in Ae. tauschii, including $Y r A S 2388^{23}$ and $Y r 28^{24}$ on $4 \mathrm{DS}^{22,25}$, and YrY201 26 on 7DL. Synthetic hexaploid wheat (SHW) lines, which contain a diversity of Ae. tauschii accessions ${ }^{27}$, are potential breeding stocks. However, many biotic and abiotic resistance genes are suppressed in the hexaploid background ${ }^{28}$. To prevent a linkage drag of undesirable traits and resistance suppression, it is best to identify $R$ genes and use them precisely in gene pyramids. In this study, we have cloned the stripe rust resistance gene YrAS2388 from Ae. tauschii. Additionally, we have demonstrated that this gene can express effectively in hexaploid wheat and barley. Deployment of YrAS2388R in wheat cultivars together with other effective genes should sustainably protect wheat production from the devastating disease wheat stripe rust.

\section{Results}

YrAS2388R confers resistance to wheat stripe rust. Aegilops tauschii CIae9, PI511383 and PI511384 (all from the subspecies (subsp.) strangulata) possess $Y r A S 2388 R^{22}$. At the two-leaf seedling (juvenile) stage, CIae9, PI511383, and/or PI511384 were resistant with infection types (IT) between 1 and 5 to nine Pst races (PSTv-3, PSTv-4, PSTv-11, PSTv-18, PSTv-37, PSTv-41, PSTv-51, PSTv-52, and PSTv-172), under low temperature (LT) and/or high temperature (HT) regimes (Table 1). These races are virulent on a wide range of wheat germplasm (Supplementary Data 1). CIae9, PI511383 and PI511384 have shown Pst resistance (IT scores =1-3; Fig. 1a) under natural infections in the Sichuan basin in China since 1995. In contrast, Ae. tauschii AS87, PI486274 and PI560536 (all subsp. tauschii accessions) do not

Table 1 Seedling responses of selected lines to Puccinia striiformis f. sp. tritici

\begin{tabular}{|c|c|c|c|c|c|c|c|}
\hline \multirow[t]{2}{*}{ Materials $^{\mathbf{a}}$} & \multirow[t]{2}{*}{ Genomes } & \multirow[t]{2}{*}{ YrAS2388R } & \multicolumn{4}{|c|}{ Infection types ${ }^{c}$} & \multirow{2}{*}{$\frac{\mathbf{T R}^{\mathbf{d}}}{\mathrm{HT} / \mathrm{LT} 1}$} \\
\hline & & & PSTV-3 & PSTv-11 & PSTv-41 & PSTV-172 & \\
\hline Clae9 & DD & $+^{b}$ & 2 & 2 & 1 & 2 & $\mathrm{HT}$ \\
\hline PI511383 & DD & + & 2 & 2 & 2 & 2 & $\mathrm{HT}$ \\
\hline AvSYr28NIL & AABBDD & + & 1 & $\overline{1}$ & 6 & 2 & $\mathrm{HT}$ \\
\hline AvS & AABBDD & $-b$ & 8 & 8 & 8 & 8 & $\mathrm{HT}$ \\
\hline Clae9 & DD & + & 2 & 2 & 2 & 2 & LT1 \\
\hline PI511383 & DD & + & 2 & 1 & 2 & 2 & LT1 \\
\hline AvSYr28NIL & AABBDD & + & 2 & 1 & 8 & 2 & LT1 \\
\hline \multirow[t]{2}{*}{ AvS } & AABBDD & - & 9 & 9 & 9 & 9 & LT1 \\
\hline & & & PSTV-4 & PSTv-18 & PSTv-51 & Fielde & LT2 \\
\hline PI511383 & DD & + & 1 & 3 & 1 & 1 & LT2 \\
\hline PI511384 & DD & + & 2 & 5 & 2 & 1 & LT2 \\
\hline PI486274 & DD & - & 8 & 9 & 8 & 8 & LT2 \\
\hline AS87 & DD & - & 8 & 8 & 8 & 8 & LT2 \\
\hline SW3 & AABBDD & + & 8 & 8 & 7 & 8 & LT2 \\
\hline SW58 & AABBDD & - & 8 & 8 & 5 & 8 & LT2 \\
\hline
\end{tabular}


a
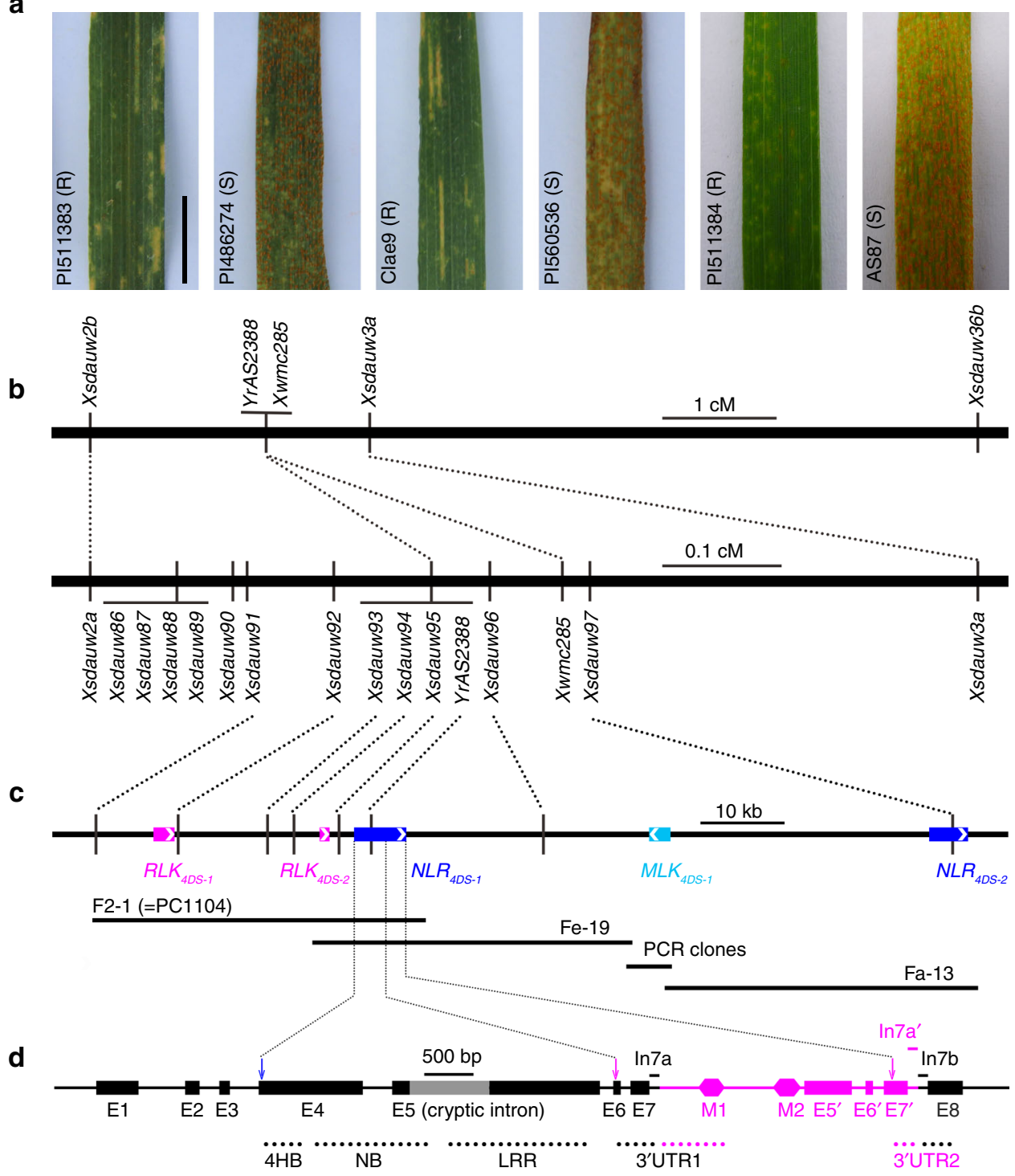

e

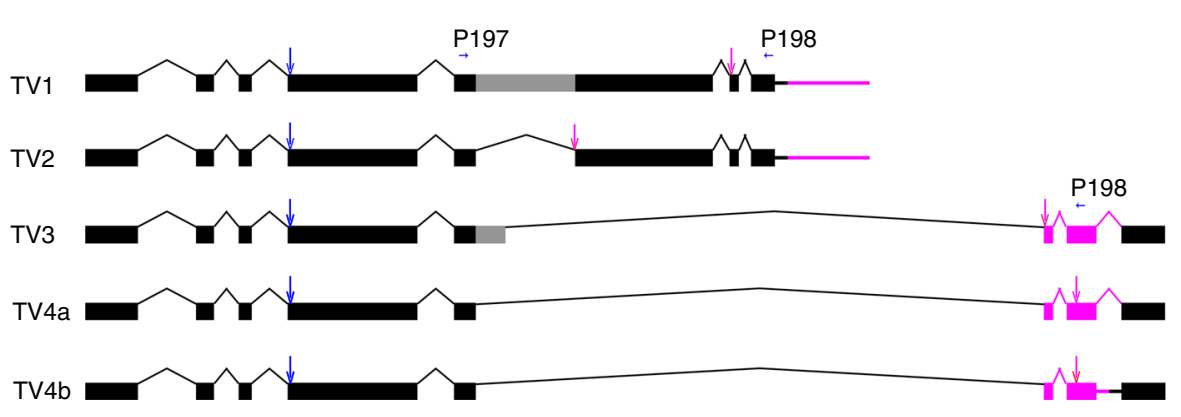

have the $\operatorname{YrAS} 2388 R$ gene $^{22}$, and were always susceptible under either natural infections or controlled inoculation (IT scores $=$ 7-9; Fig. 1a, Table 1).

Previously, we hypothesized that YrAS2388 and Yr28 are the same gene ${ }^{22}$. AvSYr28NIL and AvS are near-isogenic lines (NILs) for the Yr28 gene. During a Pst test with PSTv-3, PSTv-11, PSTv41, and PSTv-172, AvS was susceptible to all races under both LT and HT, but AvSYr28NIL was highly resistant to PSTv-3, PSTv11, and PSTv-172 (Table 1). We additionally tested two synthetic hexaploid wheat (SHW) lines, SW3 and SW58, that were derived from the durum wheat Langdon but have different D-genome donors: the Pst-resistant CIae9 and the Pst-susceptible AL8/78, respectively. Despite a dominant YrAS2388R gene in CIae9, SW3 was highly susceptible to PSTv-4, PSTv-18, PSTv-37 and PSTv-52 (Table 1), which was comparable to SW58 under LT. Thus, YrAS2388R can be suppressed when it is introgressed into certain hexaploid wheat genotypes.

YrAS2388R was delimited to a $50-\mathrm{kb}$ region in PI511383. CIae9, PI511383, PI511384 and eleven other accessions were 
Fig. 1 Map-based cloning of the YrAS2388 gene. a Adult plant responses ( $R=$ resistant; $S=$ susceptible) of parental lines to natural spores in the field. Scale bar $=1 \mathrm{~cm}$. b Genetic maps are based on popC-1 (upper) and popA-2 (lower). c Physical maps are based on three fosmid clones: F2-1 (= PC1104), Fe-19 and Fa-13, which contain five genes (colored rectangles; arrows pointing to $3^{\prime}$ ends) that encode for two 4-helix bundle-nucleotide binding-leucine rich repeat (NLRs), a malectin-like kinase (MLK), and two receptor-like kinases (RLKs). A 3.9-kb physical gap between Fe-19 and Fa-13 was closed by sequencing PCR clones. d Genomic structure of NLR $4 D S-1$ in PI511383. The conserved domains and the duplicated 3'UTRs are labelled; their approximate genomic locations are highlighted with dotted lines. The 3'UTR duplication was caused by a 2668-bp insertion (magenta region), which has three regions (with a prime symbol) similar to exons 5, 6, and 7. Introns 7a and 7b, and exon 8 are the original components of the ancestral 3'UTR, but the 2668-bp insertion disrupted the ancestral 3'UTR and then formed two 3'UTRs, each containing both ancestral (black dots) and inserted (magenta dots) segments. The cryptic intron in exon 5 is highlighted by a gray box. Introns $7 \mathrm{a}, 7 \mathrm{a}^{\prime}$, and $7 \mathrm{~b}$ have a size bar below their names. e Transcript variants of $N L R_{4 D S-1}$ in accession PI511383. Cloning and sequencing of the NLR $4 D S-1$ CDNA clones identified five transcript variants, designated TV1 to TV4b, of NLR $4 D S-1$ in

accession PI511383. Grey boxes indicate portions of the retained intron in mature messenger RNA. Rectangles and straight lines indicate regions present in mRNA; the caret-shaped lines represent regions that are absent in mRNA. Part of the cryptic intron in exon 5 is retained in TV3. TV4a and TV4b encode an identical protein, called TV4. P197 and P198 are primers that detect all five splicing variants in one PCR. Abbreviations include exon (E), four-helical bundle $(4 \mathrm{HB})$, intron (In), leucine-rich repeat (LRR), two miniature inverted-repeat transposable elements (M1 and M2), nucleotide-binding (NB), and start (downwards arrows in blue) and stop (downwards arrows in magenta) codons. Source data of Fig. 1a are provided as a Source Data file

found to have the YrAS2388R gene or allelic genes on $4 \mathrm{DS}^{22}$. We previously developed three $\mathrm{F}_{2}$ populations: popA (PI511383/ PI486274), popB (CIae9/PI560536) and popC (PI511384/AS87). The YrAS2388R-based Pst responses are inherited as a Mendelian trait in all three $\mathrm{F}_{2}$ populations ${ }^{22}$. Here, among $1910 \mathrm{~F}_{2}$ plants of popC-2 (PI511384/AS87), 1,432 were resistant and 478 were susceptible in Wenjiang, Sichuan, China, which fits single dominant gene inheritance (Chi-Square goodness of fit test, $\chi^{2}{ }_{3: 1}$ $=0.001, P=0.98$ ).

Using the wheat $10 \mathrm{k}$ iSelect array ${ }^{29}$, we genotyped CIae9, PI486274, PI511383, PI560536, and 17 Pst-susceptible $\mathrm{F}_{2}$ plants (10 from popA and 7 from popB) for bulked segregant analysis (BSA) of the Pst-susceptible allele of YrAS2388 (YrAS2388S). Among 3276 applicable single nucleotide polymorphisms (SNPs), we selected 20 SNPs that were mostly associated with a Pstsusceptible phenotype; eight of them, including AT4D3406, AT4D3410, AT4D3411, AT4D3412, AT4D3413, AT4D3417, AT4D3418, and AT4D3419 (Supplementary Table 1), were in the $4 \mathrm{DS}$ distal region ${ }^{29}$. Based on specific genotypes per marker per plant, YrAS2388S was mapped distal to the AT4D3406 region (Supplementary Table 1).

The AT4D3406 region (Supplementary Fig. 1a) was initially targeted to map the YrAS2388 gene in popA and popC. Using the $\mathrm{F}_{2}$ and $\mathrm{F}_{3}$ data, we mapped $\mathrm{YrAS} 2388$ to the same region in popA-1 and popC-1 (Supplementary Fig. 1b, c). In popC-1, YrAS2388 is between Xsdauw2b (= AT4D3403) and Xsdauw3a (= AT4D3405) (Supplementary Table 2), an approximate 2.4-cM interval (Supplementary Fig. 1c). To assure that we defined the correct region, we targeted a large interval, Xsdauw2a (= AT4D3403)-Xsdauw36a (= AT4D3410), for screening recombinants in popA-2. Additional markers were designed from the linkage map ${ }^{29}$ and genome sequence ${ }^{30}$ of Ae. tauschii. First, we retrieved the AT4D3403, AT4D3404 and AT4D3405 corresponding genomic sequence ${ }^{29}$, prioritized the low-copy number regions, created nine PCR markers (Xsdauw86 to Xsdauw91, Xsdauw93, Xsdauw95 and Xsdauw97) among six parental lines, and placed YrAS2388 between Xsdauw91 and Xsdauw97 (Supplementary Fig. 1d). Second, we constructed a fosmid genomic library from the Pst-resistant genotype PI511383. Xsdauw92, Xsdauw94 and Xsdauw96 were then developed using the fosmid clones of the YrAS2388 region. After analyzing 4205 popA $\mathrm{F}_{3}$ plants, which were from $11 \mathrm{~F}_{2}$ plants heterozygous in the $X s d a u w 2 a-X s d a u w 36 a$ region, we precisely mapped YrAS2388 between Xsdauw92 and Xsdauw96, about a 0.13-cM interval, and added to the YrAS2388 interval with three completely linked markers (Xsdauw93-Xsdauw95) (Fig. 1b, Supplementary Fig. 1d, Supplementary Table 2).
The fosmid genomic library of PI511383 has approximately one million clones and represents an eight-fold coverage of the Ae. tauschii genome $\left(\approx 4.3 \mathrm{~Gb}^{30}\right)$. Twenty fosmid clones were identified in the YrAS2388 region. In the physical map (Fig. 1c, Supplementary Fig. 2), Xsdauw91 and Xsdauw96, which delimited the YrAS2388 gene, were anchored to the two overlapping fosmids, F2-1 and Fe-19. After sequencing F2-1 and Fe-19, we primarily analyzed the Xsdauw91-Xsdauw96 region (ca. $50 \mathrm{~kb}$ ). RNA sequencing (RNA-seq) in PI511383 revealed three active genes in the Xsdauw91-Xsdauw96 interval, including two receptor-like kinase genes ${ }^{31}\left(R L K_{4 D S-1}\right.$ and $\left.R L K_{4 D S-2}\right)$, and a classic $R$ gene, $N L R_{4 D S-1}$. Both $R L K_{4 D S-1}$ and $R L K_{4 D S-2}$ are wallassociated receptor kinases. $R L K_{4 D S-1}$ has an $\mathrm{N}$-terminal galacturonan-binding and a C-terminal serine/threonine kinase (STK) domain, whereas $R L K_{4 D S-2}$ has only the STK domain. $N L R_{4 D S-1}$ has a classic four-helix bundle $(4 \mathrm{HB})$ that was previously classified as a coiled-coil, a NB domain and a LRR domain with 12 or more leucine-rich repeats. All three genes are highly conserved among the Ae. tauschii accessions tested and the collinearity of the YrAS2388 region is conserved between Ae. tauschii and common wheat (Supplementary Fig. 2). Transcription of $R L K_{4 D S-1}, R L K_{4 D S-2}$ and $N L R_{4 D S-1}$ was confirmed by reverse-transcription PCR (RT-PCR) (Supplementary Fig. 3a, b). Genome sequence analysis, RNA-seq and RT-PCR further revealed that the $N L R_{4 D S-1}$ gene in PI511383 contains a 2668bp insertion, which resulted in duplicated $3^{\prime}$ untranslated regions (3'UTR1 and 3'UTR2) and five transcript variants (Fig. 1d, e). However, the $N L R_{4 D S-1}$ gene in AL8/78 lacks the 2668-bp insertion and has only one transcript product.

The closest distal marker, Xsdauw92, placed $R L K_{4 D S-1}$ outside of the YrAS2388 interval. Consequently, $N L R_{4 D S-1}$ and $R L K_{4 D S-2}$

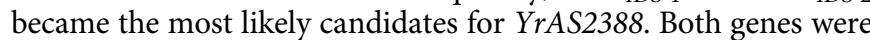
expressed in the Pst-resistant parents (CIae9, PI511383 and PI511384) and in two Pst-susceptible genotypes (AL8/78 and AS87), but were inactive in two Pst-susceptible parents (PI486274 and PI560536) (Supplementary Fig. 3a, b). In a comparison of NLR $R_{4 D S-1}$ in the Pst-resistant (CIae9, PI511383 and PI511384) and the Pst-susceptible (AS87 and AL8/78) genotypes, the cDNA and protein sequences are only $94 \%$ and $91 \%$ identical, respectively (Supplementary Data 2). The Pst-susceptible genotypes (AL8/78 and AS87) had a premature stop codon in $R L K_{4 D S-2}$, which was absent in the Pst-resistant parents (CIae9, PI511383 and PI511384). Thus, both $N L R_{4 D S-1}$ and $R L K_{4 D S-2}$ remained as candidates for the YrAS2388 gene.

Haplotype markers indicated that $N L R_{4 D S-1}$ is $\operatorname{YrAS2388}$. To help identify the correct gene, we genotyped 159 Ae. tauschii 
a

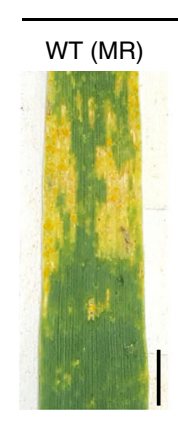

b

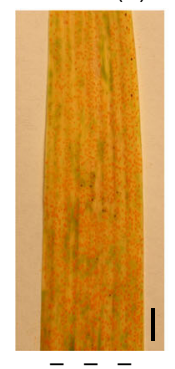

$\begin{aligned} & - \\ & -\end{aligned}-$

c $\operatorname{GDP}(\mathrm{S})$

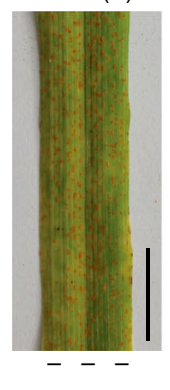

Syn-SAU-93

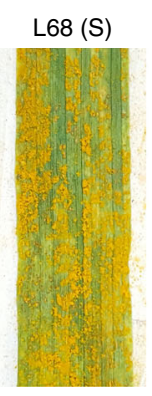

$5-6-13-5(R)$

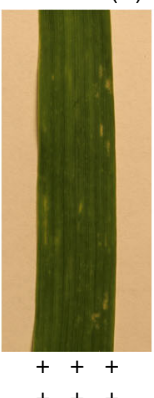

34-9 (S)

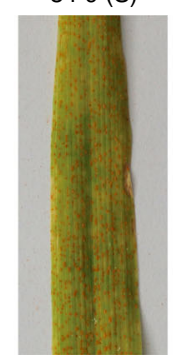

$--$

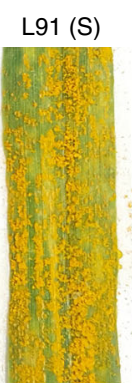

$5-6-9-29(R)$

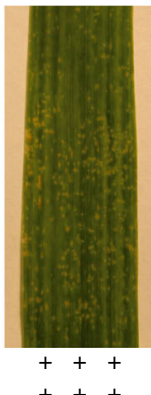

34-15 (R)

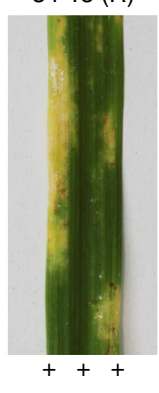

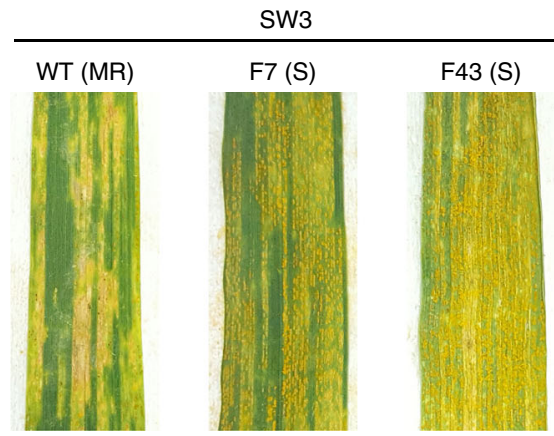

10-6-3-28 (R)

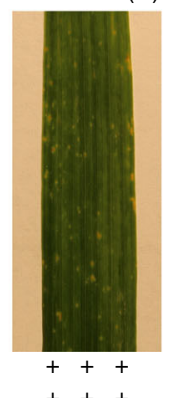

20-11 (S)

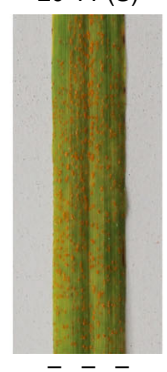

-
--

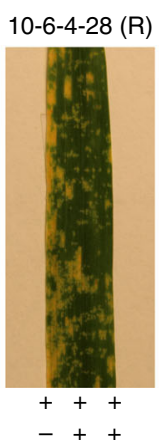

20-3 (R)

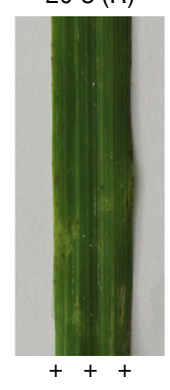

$10-6-2-30(R)$

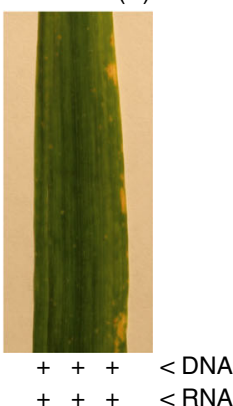

20-14 (R)

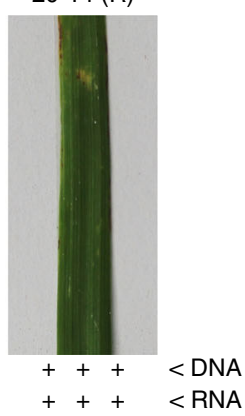

Fig. 2 The YrAS2388 locus confers stripe rust resistance in wheat and barley. a Syn-SAU-93 and SW3 are two synthetic hexaploid wheat (SHW) lines that express the YrAS2388R gene. WT is the resistant wild-type control with necrotic lesions. L68 (G117D), L91 (V267I), F7 (S394N) and F43 (V482I) are susceptible mutants with sporulating Pst. Plant responses ( $M R=$ moderate resistance; $\mathrm{R}=$ resistant; $\mathrm{S}=$ susceptible) to Pst are indicated in parentheses. b The susceptible hexaploid wheat CB037 was transformed with the intact PC1104 (=F2-1). Transgenic $T_{3}$ wheat (all from the No. 5 and $10 T_{2}$ subfamilies) was challenged with PSTv-239 at the adult plant stage. Under each picture, PCR results as positive (plus signs) or negative (minus signs) for DNA amplification (upper) and RNA expression (lower) of the three target genes: $R L K_{4 D S-1}$ (left), RLK $4 D S-2$ (middle) and NLR $4 D S-1$ (right). RT-PCR is illustrated in Supplementary Fig. 4. c The susceptible barley Golden Promise (GDP) was transformed with the intact PC1104 (=F2-1). Transgenic $T_{1}$ barley seedlings were inoculated with race PSH-72 of Puccinia striiformis $\mathrm{f}$. sp. hordei $(P s h)$. Scale bar $=1 \mathrm{~cm}$. Source data are provided as a Source Data file

accessions using five markers for $N L R_{4 D S-1}$ (HTM3a to HTM3e, or collectively called HTM3S), one for $R L K_{4 D S-1}$ (HTM1a) and one for $R L K_{4 D S-2}$ (HTM2a) (Supplementary Tables 2 and 3, Supplementary Data 3). The R-type allele (e.g. "A" in PI511383) of $N L R_{4 D S-1}$ was completely associated with Pst resistance in resistant haplotypes R1 to R3 (Supplementary Data 3). All non-A scores of the NLR $4 D S-1$ markers were associated with Pst susceptibility. The coding region (ATG to $3^{\prime} \mathrm{UTR} 2$; Fig. 1d) of $N L R_{4 D S-1}$ is identical amongst eight Pst-resistant Ae. tauschii accessions, including AS2386, AS2387, AS2399, AS2402, CIae9, PI349037, PI511383, and PI511384. In contrast, in $R L K_{4 D S-1}$ and $R L K_{4 D S-2}$, the $R$-type allele (e.g. "A" in PI511383) was present in the Pst-susceptible genotypes (S1-S3 and S5), indicating that both genes do not confer Pst resistance. Similarly, the absence of $R L K_{4 D S-1}$ and/or $R L K_{4 D S-2}$ in the $\mathrm{R} 2$ and $\mathrm{R} 3$ haplotypes suggested that neither gene is essential for Pst resistance. Thus, $N L R_{4 D S-1}$ is the only candidate for $\operatorname{YrAS} 2388 R$.
Pst-susceptible SHW mutants have more mutations in $N L R_{4 D S-1}$. Synthetic hexaploid wheat (SHW) SW $3^{32}$ and Syn-SAU-93 $3^{33}$ acquire the YrAS2388R gene from their D-genome donor; both SW3 and Syn-SAU-93 displayed moderate Pst resistance (IT scores =3-5; Fig. 2a) in Sichuan, China. Using ethyl methanesulfonate (EMS), we generated $1132 \mathrm{M}_{2}$ families of SW3 and $613 \mathrm{M}_{2}$ families of Syn-SAU-93. Under field conditions, we identified 103 Pst-susceptible plants (IT scores =7-9; Fig. 2a, Supplementary Data 4). For the $N L R_{4 D S-1}, R L K_{4 D S-1}$, and $R L K_{4 D S-2}$ genes, 51 Pst-susceptible mutants $(49.5 \%)$ had a deletion in the $N L R_{4 D S-1}$ gene, of which 50 deletion events extended into $R L K_{4 D S-2}$ but only 11 deletion events extended further to $R L K_{4 D S-1}$ (Supplementary Data 4). However, no deletion only occurred in $R L K_{4 D S-1}$ and/or $R L K_{4 D S-2}$. Among the remaining 52 non-deletion mutants, 18 Pst-susceptible mutants had at least one base change in the $N L R_{4 D S-1}$ gene, and 16 of those mutations (89\%) either caused an amino acid change or formed a premature stop codon (Supplementary Data 4). 
Table 2 Transgene expression and plant responses to Puccinia striiformis

\begin{tabular}{|c|c|c|c|c|c|c|}
\hline Groups $^{a}$ & Constructs (treatment) $b$ & Events ${ }^{c}$ & $R L K_{4 D S-1}$ & $R L K_{4 D S-2}$ & $N L R_{4 D S-1}{ }^{d}$ & Responses to Pst (or Psh)e \\
\hline G2 & PC1104 (X1) & 1 & + & + & - & Susceptible \\
\hline G3 & PC1104 (I) & 1 & $-d$ & + & + & Resistant \\
\hline G5 & PC1104 (B1, N1, XK1) & 5 & - & + & - & Susceptible \\
\hline G6 & PC1104 (B1, N1, X1) & $7+2$ & - & - & - & Susceptible \\
\hline G7 & PC1101 (Ubi::NLR $4 D S-1 T V 1)^{f}$ & $9+4$ & - & - & + & Susceptible \\
\hline
\end{tabular}

aGroups G1-G6 are based on genomic DNA, and Groups G7-G8 are based on cDNA

bPC1104 was either intact (I) or linearized with BsrGI (B1), NotI (N1), Xbal (X1) and Xbal plus Kpnl (XK1). Intact or linearized plasmid per enzyme was introduced into recipient genotypes separately 'Per cell, the first number indicates the number of wheat transformants; when there are two numbers, the second number indicates the number of barley transformants

${ }^{\mathrm{d}} \mathrm{A}$ plus sign = positive for full-length gene expression by PCR; a minus sign = negative for gene expression. RT-PCR is illustrated in Supplementary Fig. 4

ePSTV-40 and PSH-72 were used to test the transgenic wheat and barley, respectively.

fThe $N L R_{4 D S-1}$ CDNAs were under the maize Ubi promoter ${ }^{68}$; no digestion was applied to them

Seven amino acid variations were identified in the $N L R_{4 D S-1}$ gene, including Gly117Asp, Val267lle, Ser394Asn, Leu421Phe, Thr456Ile, Val482Ile, and Gln557Stop $\left(^{*}\right)$ (Supplementary Data 4). However, all 52 non-deletion mutants had no mutation in the coding region of $R L K_{4 D S-2}$. Thus, $N L R_{4 D S-1}$ confers resistance to Pst in SW3 and Syn-SAU-93 and likely represents the YrAS2388R gene.

$N L R_{4 D S-1}$ confers stripe rust resistance in wheat and barley. We transformed wheat and barley with the fosmid PC1104 (= F2-1; Supplementary Table 4), which has a $40-\mathrm{kb}$ genomic fragment with $N L R_{4 D S-1}, R L K_{4 D S-1}$ and $R L K_{4 D S-2}$. The spring wheat CB037 34 was selected as the primary wheat recipient genotype because it is highly susceptible (IT scores $=7-9$ ) to 11 Pst races, including PSTv-4, PSTv-14, PSTv-37, PSTv-39, PSTv-40, PSTv-47, PSTv143, PSTv-221, PSTv-239, PSTv-306, and PSTv-353 (Supplementary Data 1). After bombarding 1,590 wheat immature embryos, we obtained 24 putative transgenic plants. $N L R_{4 D S-1}$, $R L K_{4 D S-1}$ and $R L K_{4 D S-2}$ were detected in four $\mathrm{T}_{0}$ plants (No. 4, 5, 10 , and 22), but only two plants (No. 5 and 10) were positive for the $N L R_{4 D S-1}$ cDNA (Table 2, Supplementary Fig. 4). For No. 5 and 10 transgenic plants, we selected 13 subfamilies that were homozygous resistant to PSTv-40 in the $\mathrm{T}_{2}$ generation, and tested the $\mathrm{T}_{3}$ plants against nine Pst races (PSTv-37, PSTv-39, PSTv-47, PSTv-143, PSTv-221, PSTv-239, PSTv-306, PSTv-352, and PSTv353) and Pst spores from the field (Supplementary Data 1). The $\mathrm{T}_{3}$ plants were resistant (IT scores $=0-4$ ) to all Pst races at the seedling and adult-plant stages (Fig. 2b, Supplementary Fig. 5, Supplementary Data 1).

Barley cultivar Golden Promise is susceptible to race PSH-72 of the barley stripe rust pathogen, P. striiformis f. sp. hordei (Psh). After bombarding 2,200 barley immature embryos with PC1104, we obtained five putative transgenic lines. Only three $\mathrm{T}_{1}$ families (No. 20, 34 and 35) segregated for their responses to PSH-72 (Table 2, Fig. 2c). $\mathrm{T}_{1}$ plants with functional $N L R_{4 D S-1}, R L K_{4 D S-1}$ and $R L K_{4 D S-2}$ were resistant (IT scores $=1-5$ ), while the ones lacking the three genes were susceptible (IT scores $=7-8$ ). Therefore, the fosmid PC1104 confers stripe rust resistance in transgenic wheat and barley.

Because PC1104 has $N L R_{4 D S-1}, R L K_{4 D S-1}$ and $R L K_{4 D S-2}$ genes, we cut PC1104 using a specific restriction enzyme (either BsrGI, $K p n \mathrm{I}, \mathrm{Not} \mathrm{I}, \mathrm{Xba \textrm {I }}$, or $K p n \mathrm{I}+\mathrm{Xba \textrm {I } )}$ to cleave/inactivate each of them, and then used the DNA fragments from each digestion to transform wheat separately (Supplementary Fig. 6). After bombarding 6,790 wheat immature embryos, we obtained 148 putative transgenic plants. Transgenic $\mathrm{T}_{1}$ and $\mathrm{T}_{2}$ plants were tested with Pst spores from the Parker Farm field (Moscow, ID, USA). Only transgenic plants that expressed $N L R_{4 D S-1}$ were resistant to Pst (Table 2, Supplementary Fig. 4). Therefore, $N L R_{4 D S-1}$ represents a strong candidate for YrAS2388 (Table 2).

The Pst-resistant $N L R_{4 D S-1}$ has duplicated $3^{\prime}$ UTRs (Fig. 1d) in all Pst-resistant parents (CIae9, PI511383 and PI511384) and each $3^{\prime} \mathrm{UTR}$ is associated with multiple transcript variants: TV1 and TV2 with 3'UTR1, TV3 and TV4a (and 4b) with 3'UTR2 (Fig. 1e). We overexpressed the Pst-resistant $N L R_{4 D S-1}$ cDNA under the maize $U b i$ promoter (Supplementary Table 4). All 36 transgenic wheat and barley lines that expressed TV1 (or TV2) did not confer resistance to stripe rust (Table 2), suggesting that one cDNA isoform was insufficient to confer stripe rust resistance. For stripe rust resistance, the $N L R_{4 D S-1}$ gene may require the activity of multiple cDNA isoforms and/or regulatory elements in the genomic sequence.

Innate and external factors regulate $N L R_{4 D S-1}$ expression. In the Pst-resistant $N L R_{4 D S-1}$, the most abundant isoforms are TV1 (for a 1068-aa protein with complete $4 \mathrm{HB}, \mathrm{NB}$, and LRR domains) and TV4 (for a 471-aa protein with a complete $4 \mathrm{HB}$ and a partial NB domain) (Fig. 1e, Supplementary Fig. 7a). The less abundant isoform TV2 might result from either a partial exon skipping from TV1 or the retention of an 833-bp cryptic intron in exon 5, which disrupts the NB and LRR domains. TV3 is also a less abundant isoform and is structurally similar to TV4, but retains the first $244 \mathrm{bp}$ in the 833-bp cryptic intron, which only disrupts the LRR domain. In contrast, the Pst-susceptible $N L R_{4 D S-1}$ either remained completely silent in PI486274 and PI560536 or produced only the TV1-type transcript in AL8/78 and AS87 (Supplementary Fig. 3b).

In Pst-resistant PI511383, TV1 to TV4 cDNAs were all expressed in the seedling and adult leaves (Supplementary Fig. 3c). When exposed to alternating low $\left(10^{\circ} \mathrm{C}\right)$ and high $\left(25^{\circ} \mathrm{C}\right)$ temperatures, the high temperature upregulated TV2 and downregulated TV4 (Supplementary Fig. 3d), which is correlated with increased Pstresistance at elevated temperatures. In response to Pst race PSTv306, the TV1 cDNA levels in the Pst-infected plants were comparable to those in the mock-inoculated control plants (Supplementary Fig. 3e). In contrast, Pst infections upregulated TV2 at 2, 5, and 10 days post inoculation (dpi) but not at $3 \mathrm{dpi}$, downregulated TV3 at 3, 7, and $14 \mathrm{dpi}$, and downregulated TV4 at 3 dpi (Supplementary Fig. 3e). Thus, both temperature and Pst infection regulate the transcription of $N L R_{4 D S-1}$. However, a change in the relative levels of either the individual four transcripts and/or the proteins or protein complexes may affect the induction of stripe rust resistance. Among the Pst-susceptible mutations of NLR 4 DS- 1 , Ser394Asn and Gln557Stop $\left(^{*}\right)$ only affect TV1 and Thr456Ile only affects TV4, which indicates that both 
TV1 and TV4 are essential for stripe rust resistance (Supplementary Data 4). Collectively, we hypothesize that TV1 plays a major role in the induction of stripe rust resistance, TV2 acts as a positive co-factor, and TV4 (or possibly TV3) act either as negative regulators when its expression is high or as positive regulators when its expression is low (Supplementary Fig. 8).

Using a yeast two-hybrid system, we tested the interaction among the native (TV1, TV2, and TV4) and mutant (TV1 G117D, TV2 ${ }^{\text {G117D }}$ and TV2V267I; Supplementary Data 4, Supplementary Fig. 7a) isoforms of the Pst-resistant $N L R_{4 D S-1}$. The NLR 4 DS-1 isoforms, both native and mutant forms (NM forms) had no autoactivity. A strong interaction occurred amongst the TV2 proteins (NM forms; Supplementary Fig. 7b). We observed a weak interaction between TV2 mutants and TV1 (NM forms), and between TV2 proteins (NM forms) and TV4. Apparently, TV2 can mediate protein interactions amongst multiple isoforms of NLR 4 DS-1.

The Pst-resistant NLR $R_{4 D S-1}$ occurs only in Aegilops tauschii. The D genome of common wheat was derived from Ae. tauschii subsp. strangulata or tauschii ${ }^{20}$. The resistance allele of $N L R_{4 D S-1}$ is present in $100 \%(n=37)$ and $19 \%(n=122)$ of the accessions of subsp. strangulata and tauschii tested, respectively (Supplementary Data 3). Similarly, the resistance allele of $N L R_{4 D S-1}$ is present in $30 \%(n=23)$ of the Ae. tauschii accessions used as a parent in developing SHW lines (Supplementary Data 5). Surprisingly, the resistance allele is absent in all $(n=461)$ of the common wheat lines tested (Supplementary Table 5, Supplementary Data 6). The $N L R_{4 D S-1}$ allele in Chinese Spring (CS) is nearly identical to the Pst-susceptible alleles from the subsp. tauschii accessions PI486274 and PI560636 (Supplementary Data 2). In addition, the resistant $N L R_{4 D S-1}$ allele is also absent in all the tested T. monococcum subsp. aegilopoides $(n=24), T$. monococcum subsp. monococcum $(n=24)$, T. turgidum subsp. dicoccoides $(n=140)$, Ae. comosa $(n=17)$, Ae. comosa var. subventricosa $(n=6)$, Ae. longissimi $(n=8)$, Ae. sharonensis $(n=28)$, Dasypyrum villosum $(n=10)$, and Hordeum vulgare subsp. spontaneum $(n=5)$ (Supplementary Table 5, Supplementary Data 6).

The Pst-resistant $N L R_{4 D S-1}$ may arise from paralogous genes. All Pst-resistant $N L R_{4 D S-1}$ genes contain two duplicated regions. The first region includes the $3^{\prime}$ end of exon 5, exons 6 and 7, and intron $7 \mathrm{a}$; and the second region includes the pseudo-exon $5^{\prime}$, exons $6^{\prime}$ and $7^{\prime}$, and intron $7 a^{\prime}$ (Fig. 1d, e, Supplementary Fig. 9a). This duplication is not present either in Pst-susceptible NLR ${ }_{4 D S-1}$ alleles or in any $N L R_{4 D S-1}$-like genes. To examine the origin of the duplicated regions, we built separate phylogenetic trees for each of six selected fragments (exons 5, 6, 7, and 8; and introns 7a and $7 \mathrm{~b}$ ) of 7 to $15 N L R_{4 D S-1}$ homologues in Triticeae (Supplementary Fig. 9b). The trees indicate that exons (5-8) and introns (7a and $7 \mathrm{~b}$ ) of the Pst-resistant $N L R_{4 D S-1}$ are more related to those of the Pst-susceptible NLR $4 D S-1$ in CS (CS-4D:1821950..1825589); all the duplicated fragments (exons $5^{\prime}$ to $7^{\prime}$ and intron $7 \mathrm{a}^{\prime}$ ) are in separate clades. In addition, the duplicated $3^{\prime}$ UTR1 and $3^{\prime}$ UTR2 DNA of $N L R_{4 D S-1}$ in PI511383 are only $87 \%$ identical in the conserved $373 \mathrm{bp}$ (GenBank MK736661: $3735 . .4107$ versus 6409..6781, counted forward from the start codon ATG). Thus, the Pst-resistant $N L R_{4 D S-1}$ likely arose after a shuffling event between two paralogous genes. Specifically, the 3'UTR2 contains part of a 2668-bp insertion (within a 6-bp target site duplication $=$ TACTGG) that occurred in intron 7 of the ancestral $3^{\prime}$ UTR1 region. A similar $3^{\prime} \mathrm{UTR}$ duplication in the Pst-resistant $N L R_{4 D S-1}$ gene is present in the synthetic wheat W7984 ${ }^{35}$. In the 2668-bp insertion, a 496-bp region (pseudo-exon exon $5^{\prime}$ ) is $90 \%$ identical to the ancestral exon 5. The insertion also has two miniature inverted-repeat transposable elements, which are frequently adjacent to transcriptionally active genes ${ }^{36}$. Likely, the 2668-bp fragment was derived from another, currently unidentified, $N L R_{4 D S-1}$ homologue in Ae. tauschii.

In Triticeae, there are multiple $N L R_{4 D S-1}$-like genes; three copies were identified in the YrAS2388 region (Supplementary Fig. 2). In common wheat $\mathrm{CS}$, there are at least five transcriptionally active homologues of the $N L R_{4 D S-1}$ gene (Supplementary Fig. 10). None of the $N L R_{4 D S-1}$-like homologues in CS has duplicated $3^{\prime}$ UTRs. The Pst-susceptible NLR $4 D S-1$ homologues in CS share only 86\%-94\% identity with the Pst-resistant NLR $4 D S-1$ in PI511383 at the cDNA level.

$N L R_{4 D S-1}$ offers a toolbox for solving stripe rust problems. We compared the stripe rust resistance in 81 SHW lines ${ }^{33}$ and their original parents, including 30 SHW lines with the YrAS2388R gene (Supplementary Data 5). YrAS2388R confers a strong Pst resistance (IT scores $=1-3$ ) in Ae. tauschii ${ }^{22}$. However, $27 \%$ of SHW wheat had significantly less resistance than the parental lines ( $T$. turgidum and/or Ae. tauschii). In this study, SW3 has the Pst-resistant $N L R_{4 D S-1}$ allele and shows the characteristic expression of alternatively spliced transcripts. However, SW3 was susceptible (IT scores =7-9) to Pst in Moscow, ID, USA (Table 1), presumably because of a suppressor in its genetic background. Nonetheless, Ae. tauschii accessions with a strong Pst resistance frequently conferred moderate to high Pst resistance in a derived SHW wheat (Supplementary Data 5), indicating that Ae. tauschii is valuable for breeding resistant $N L R_{4 D S-1}$. For example, the SHW wheat Syn-SAU-S9 is based on Langdon/AS313//AS2399, in which the Ae. tauschii AS2399 is positive for the YrAS2388R gene ${ }^{22}$. Although SynSAU-S9 displayed only moderate resistance to Pst (IT scores = 4-5), we used Syn-SAU-S9 to transfer the YrAS2388R gene into common wheat. Three co-segregating markers were used for marker-assisted selection of YrAS2388R (Supplementary Fig. 11, Supplementary Table 2). In 2015, we developed an elite line Shumai 1675, which is an $\mathrm{F}_{6}$ line of Syn-SAU-S9/Chuan 07001//Shumai 969. Shumai 1675 is highly resistant to Pst in Sichuan, China. In 2017, Shumai 1675 outcompeted the check variety Mianmai 367 with an $11 \%$ increase in yield in the regional variety trials of the Sichuan province, China (Supplementary Table 6).

\section{Discussion}

YrAS2388R provides robust resistance in a wide spatial and temporal range, including China (current study), Canada ${ }^{37}$, Norway $^{25}$, the United Kingdom ${ }^{38}$ and the United States (TA2450 = CIae9, TA2452 = PI511384 ${ }^{39}$; current study). However, YrAS2388R has had limited use probably for two reasons: it is absent in common wheat; and it can be suppressed in hexaploid wheat. In the present study, $\operatorname{YrAS} 2388 R$, when separated from potential linkage drag, conferred strong stripe rust resistance in transgenic wheat and barley, indicating that $\operatorname{YrAS} 2388 \mathrm{R}$ offers a practical solution for stripe rust resistance in Triticeae. The YrAS2388R gene-based markers (e.g. Xsdauw95, Supplementary Fig. 11) can be used for marker-assisted selection.

YrAS2388R is another example of a gene that was either not transferred or lost during domestication. Nevertheless, genes from both progenitors and distantly-related species of wheat can be used to enhance contemporary common wheat. Of the 81 permanently named $\mathrm{Yr}$ resistance genes, 21 were transferred from either related species or wild relatives of wheat, such as $\operatorname{Yr} 5$ from Triticum spelta, Yr15 and Yr36 from T. dicoccoides and Yr28 from Ae. tauschii ${ }^{40}$. However, alien genes can be accompanied by linkage drag ${ }^{41}$. For example, linked genes to $\mathrm{Yr} 8$ from Ae. comosa 
are associated with tall height and delayed maturity ${ }^{42}$. The $\operatorname{Yr} 9$ gene from the 1BL/1RS translocation improves grain yield but causes inferior quality ${ }^{43}$, which limits its use in wheat especially in the U.S. Pacific Northwest ${ }^{44}$. YrAS2388R could be transferred into wheat through a cisgenic approach. Thus, cisgenic YrAS2388R can provide an advantage to consumers in comparison to traditional breeding.

YrAS2388R (or Pst-resistant $N L R_{4 D S-1}$ ) is associated with duplicated $3^{\prime}$ UTRs, which is an apparently rare phenomenon. The ancestral $3^{\prime} U T R$ of $N L R_{4 D S-1}$ adjoined the $3^{\prime}$-end of an unknown $N L R_{4 D S-1}$ paralog, resulting in duplicated $3^{\prime}$ UTRs in Pst-resistant $N L R_{4 D S-1}$. The $3^{\prime} \mathrm{UTR}$ is an important component of eukaryotic genes ${ }^{45}$. More than half of human genes use alternative polyadenylation to generate mRNAs that differ in the $3^{\prime}$ UTR length but encode the same protein ${ }^{46}$. In contrast, there are few reports of genes with two separate $3^{\prime}$ UTRs that cause a difference in the protein product. In wheat, the stripe rust resistance gene WKS1 generated six transcript variants, of which WKS1.1 differs from the others in the $3^{\prime} \mathrm{UTRs}^{14}$. Pst-resistant $N L R_{4 D S-1}$ also shows alternative splicing (AS) in the NB-LRR region of the gene. AS is prevalent in eukaryotes ${ }^{47} ; 95 \%$ of multi-exon genes in human ${ }^{48}$ and $44 \%$ of multi-exon genes in Arabidopsis ${ }^{49}$ display AS. In Arabidopsis, the bacterial-resistance gene RPS4 produces alternative transcripts in response to infection by pathogen Pseudomonas syringae pv. tomato ${ }^{50}$. Both environmental and developmental stimuli precisely regulate the abundance of functional mRNA isoforms ${ }^{51}$. Here, in keeping with resistance, expression of the $N L R_{4 D S-1}$ isoforms also depends on pathogen infection and the temperature. Thus, abundance of $N L R_{4 D S-1}$ isoforms appears to be a mechanism that wheat can use to robustly resist stripe rust pathogen invasion.

The NLR $_{4 \mathrm{DS}-1}$ protein is a member of the CC-NB-LRR (CNL) proteins. The coiled-coil domain of the potato virus $\mathrm{X}$ resistance protein $(\mathrm{Rx})$ actually forms a four-helix bundle $(4 \mathrm{HB})^{52}$. The N-terminal domain of $\mathrm{NLR}_{4 \mathrm{DS}-1}$ is predicted to fold into four helixes, and it is also classified as an Rx-CC-like in the NCBI CDD $\left(E=9 \times 10^{-9}\right)$ and $\mathrm{Rx} \_\mathrm{N}$ in the Pfam database $\left(\mathrm{E}=6 \times 10^{-16}\right)$. Although $\mathrm{CNL}$ genes are often race-specific and not durable ${ }^{53}$, some CNL genes such as the rice blast resistance gene Pigm $R^{54}$ have been durable. Here, we showed that $\operatorname{YrAS} 2388 \mathrm{R}$ confers resistance to a broad array of Pst races and has been effective to all natural infections of Pst in China since 1995. As a typical NLR gene, we hypothesize that the $\mathrm{NLR}_{4 \mathrm{DS}-1}$ proteins change their state via a competition model (Supplementary Fig. 8). The full-length TV1 protein plays a central role in signal transduction, but it requires other variant proteins (TV2 and TV4) for a proper conformation, which together form an active TV1 complex for defense signaling.

Here, YrAS2388R was fully expressed without suppression in transgenic hexaploid wheat and in barley. In addition, we have produced Shumai 1675, which has $\operatorname{YrAS2388R}$ and is strongly resistant to Pst, suggesting that either $Y r A S 2388 R$ is not suppressed in Shumai 1675 or that $\operatorname{YrAS} 2388 R$ worked positively with other $Y r$ genes to confer resistance to Pst. However, in the current study, the resistance levels of parental lines (T. turgidum and/or Ae. tauschii) were suppressed in nearly $27 \%$ of the SHW wheat lines. Yr28, which is probably the same gene as YrAS2388 22 , was effective in seedlings and adult plants of SHW Altar 84/Ae. tauschii accession W-21924. Here, we observed that YrAS2388R in SHW SW3 was suppressed, i.e., it was fully susceptible to natural Pst races at adult-plant stages in Moscow (ID, USA), probably because the suppressor responds more to the cooler night temperatures in this area. When YrAS2388R is suppressed in a specific hexaploid wheat such as SW3, Pstresistance levels might be increased by disrupting the unknown suppressor, as was previously done by inactivating a suppressor of stem rust resistance ${ }^{55}$.

In the case of wheat powdery mildew, pyramiding of closely related NLR genes can cause dominant-negative interactions and that lead to $R$ gene suppression ${ }^{56}$. For example, the $P m 8$ resistance gene from rye was suppressed in wheat by a susceptible allele of the wheat ortholog $P m 3^{57}$. In the present study, the Pstresistant $N L R_{4 D S-1}$ in PI511383 shares $86-94 \%$ identity with cDNA from the transcriptionally active homologues in common wheat (Supplementary Fig. 10). Thus, YrAS2388R suppression might conceivably be caused by close homologues of $N L R_{4 D S-1}$ that are present in Triticeae. To test this hypothesis, in the future, one could mutagenize a SW3 line, screen for truncation mutations in the $N L R_{4 D S-1}$ homologues, and test whether the homologues' mutations have any effect on stripe rust resistance. Regardless, because the transgene $N L R_{4 D S-1}$ induces effective Pst resistance in hexaploid wheat, we predict that sustainable Pst resistance can be achieved with either a cisgenic strategy with Pst-resistant $N L R_{4 D S-1}$ or a conventional strategy that combines both the incorporation of a Pst-resistant $N L R_{4 D S-1}$ and either avoidance or inactivation of the apparently linked latent suppressor(s) from Ae. tauschii.

\section{Methods}

Plant materials. This study was performed on Aegilops tauschii, Hordeum vulgare, Triticum aestivum and synthetic hexaploid wheat $\left(\mathrm{SHW}^{58}\right)$ (Supplementary Table 7) Sources of accessions used for haplotype analysis are indicated in Supplementary Data 3 and 6. To map YrAS2388, we used six Ae. tauschii accessions (Supplementary Table 7), in which the Pst-resistant parents, CIae9, PI511383 and PI511384 (= AS2388), all have YrAS2388R 22 .

We developed three $F_{2}$ populations (popA: PI511383/PI486274; popB: CIae9/ PI560536; and popC: PI511384/AS87 ${ }^{23}$ ). These populations were used for preliminary and fine mapping, and popC was also used to confirm the single Mendelian inheritance of $Y r A S 2388$. In popA, we selected $11 \mathrm{~F}_{2}$ plants that were heterozygous in the YrAS2388 region (Xsdauw2-Xsdauw36), and allowed them to self-pollinate to produce $\mathrm{F}_{3}$ seeds. After screening 4,205 $\mathrm{F}_{3}$ plants, we identified 467 plants with crossovers in the Xsdauw2-Xsdauw36 interval, and used them to generate a high-density map.

Stripe rust inoculum and infection assays. Wheat stripe rust tests were conducted in four institutions: Shandong Agricultural University (SDAU), Tai'an, China; Sichuan Agricultural University (SCAU), Chengdu, China; Washington State University (WSU), Pullman, USA; and University of Idaho (UI), Moscow, USA. Avocet Susceptible (AvS), Huixianhong, Mingxian 169, and/or SY95-71 were used as susceptible checks and also planted surrounding the plots to increase and spread urediniospores for adequate and uniform rust levels for reliable screening. For winter-growth genotypes tested in greenhouses or growth chambers, seeds were vernalized in wet germination paper (Anchor Paper Co., Saint Paul, MN, USA) at $4^{\circ} \mathrm{C}$ in darkness for $45 \mathrm{~d}$; vernalized shoots were transplanted into soil in the greenhouse and maintained at $25^{\circ} \mathrm{C}$ during the day and $15^{\circ} \mathrm{C}$ at night with $16 \mathrm{~h}$ photoperiod.

Infection types (IT) were recorded using a $0-9$ scale $^{59}$ and the following categories: resistant $(\mathrm{R}, \mathrm{IT}$ scores $=0-3)$, moderate reactions $(\mathrm{M}, \mathrm{IT}$ scores $=$ 4-6) that include moderate resistance (MR, IT scores $=4-5)$ and moderate susceptibility (MS, IT score $=6$ ), and susceptible (S, IT scores $=7-9$ ). IT scores were recorded 15-18 days post inoculation (dpi) when the uredinial pustules were clearly visible on susceptible plants. Responses of SHW and their parental lines to Pst are shown in Supplementary Data 5.

At SDAU, urediniospores were obtained from the Institute of Plant Protection, Chinese Academy of Agricultural Sciences, Beijing, China. Due to changes in race frequency and spore availability, different $P$ st races were used in different years (mixed spores of Chinese Pst races CYR29, CYR31, CYR32, CYR33, Su11 and/or Su14 during 2010 to 2012; CYR29 and CYR32 in 2013; and CYR29, CYR31, CYR32 and CYR33 in 2014-2016). Collectively, these races represent the predominant Pst races in China in different periods since the 1990's. Field trials were performed to assess the responses to Pst in the parental lines, $\mathrm{F}_{1}, \mathrm{~F}_{2}$ and advanced progenies of popA and popB. At the seedling stage, an aqueous spore suspension was manually injected with a $2.5 \mathrm{ml}$ syringe into leaf bundles and repeated after 10 days. For preliminary mapping, $\mathrm{F}_{2}$ plants of popA and popB were evaluated in 2011, and the corresponding $\mathrm{F}_{3}$ progeny were then tested in 2012. Critical recombinants of popA were evaluated in 2013-2016 ( $\mathrm{F}_{3}$ to $\mathrm{F}_{6}$ generations, one generation per year), and the $\mathrm{F}_{4}-\mathrm{F}_{6}$ generations were additionally tested in SCAU in 2014-2016.

At SCAU, we primarily conducted the Pst test in Dujiangyan and Wenjiang, two experimental stations of the Triticeae Research Institute at SCAU.

Urediniospores were obtained from the Research Institute of Plant Protection, 
Gansu Academy of Agricultural Sciences, Lanzhou, China. Using the mixture of Chinese Pst races CYR30, CYR31, CYR32, SY11-4, SY11-14, and HY46-8, we evaluated the Ae. tauschii germplasm in Dujiangyan for three growing seasons (2006-2009). In 2008-2009, we also tested synthetic wheat and their polyploid parents in Dujiangyan (Supplementary Data 5). Using a mixture of CYR30, CYR31, CYR32, CYR33, SY11-4 and HY46-8, we retested synthetic wheat and their parent lines in Wenjiang in 2011-2012 (Supplementary Data 5) 22, and then retested five synthetic wheat and their parent lines in Wenjiang in 2016-2017 using a mixture of CYR32, CYR33, CYR34 (= Gui22-9), Gui22-14, and SY11-4 (Supplementary Data 5). To identify Pst-susceptible mutants, we screened the Syn-SAU-93 population in 2016-2018 and the SW3 population in 2017-2018 using urediniospores of similar races as 2016-2017. For popC, we assessed the parental lines, $F_{1}, F_{2}$ and advanced progenies from 2010 to 2016. In 2011, $F_{2}$ plants were tested in Wenjiang, and the field plots were inoculated at $7 \mathrm{wk}$ after planting with a mixture of CYR30, CYR31, CYR32, CYR33, SY11-4 and HY46-8.

At WSU, urediniospores were produced by the USDA-ARS Wheat Unit at Pullman, WA, USA. The plants were initially grown in a greenhouse at 15 to $25^{\circ} \mathrm{C}$. At the two-leaf stage, we prepared a mixture of urediniospore and talc at 1:20 ratio ( $v$ vs. v), dusted it on plants, and then applied a water mist onto the plants. The inoculated plants were incubated in a dew chamber at $10^{\circ} \mathrm{C}$ in the dark for $24 \mathrm{~h}$, and then moved to growth chambers for either low or high temperature tests. The low temperature (LT) cycle had a 16-h photoperiod ( 6 a.m.-10 p.m.) with a diurnal temperature cycle of $4{ }^{\circ} \mathrm{C}$ at 2 am and a gradual increase to $20^{\circ} \mathrm{C}$ at $2 \mathrm{pm}$ followed by a gradual decrease to $4^{\circ} \mathrm{C}$ at $2 \mathrm{am}$. The high temperature (HT) cycle had a $16-\mathrm{h}$ photoperiod with a gradual temperature gradient from $10^{\circ} \mathrm{C}$ at 2 a.m. to $30^{\circ} \mathrm{C}$ at 2 p.m. and then back to $10^{\circ} \mathrm{C}$ at 2 a.m.

At UI, urediniospores were produced by the USDA-ARS Wheat Unit at Pullman, WA, USA. Transgenic plants and wild-type controls were grown in chambers. At either the two-leaf stage for seedlings or at the 6-leaf stage for adults, plants were dust-inoculated using the urediniospore and talc mixture (1:20), maintained at $10^{\circ} \mathrm{C}$ for $48 \mathrm{~h}$ in dark, and then maintained under a modified LT cycle $\left(10^{\circ} \mathrm{C}\right.$ for $12 \mathrm{~h}$ with 8 -h of darkness in the middle, $20^{\circ} \mathrm{C}$ for $8 \mathrm{~h}$ in the middle of 16-h light, with a gradual transition from 10 to $20^{\circ} \mathrm{C}$ within a 2-h light period and vice versa for a gradual transition from 20 to $10^{\circ} \mathrm{C}$ ) or under a modified HT cycle $\left(15^{\circ} \mathrm{C}\right.$ for $12 \mathrm{~h}$ with 8 -h darkness in the middle, $25^{\circ} \mathrm{C}$ for $8 \mathrm{~h}$ in the middle of a 16-h light, with a gradual transition from 15 to $25^{\circ} \mathrm{C}$ within a 2-h light period, and vice versa for a gradual transition from 25 to $15^{\circ} \mathrm{C}$ ).

Bulked segregant analysis of the YrAS2388 gene. Genomic DNA was extracted using the Sarkosyl method ${ }^{17}$. Infinium iSelect genotyping was assayed at the Genome Center (University of California, Davis, CA, USA). Normalized Cy3 and Cy5 fluorescence for each DNA sample was plotted with the GenomeStudio program (Illumina, Inc., San Diego, CA, USA), resulting in genotype clustering for each SNP marker ${ }^{20}$.

We performed bulked segregant analysis (BSA) on four parents (CIae9, PI486274, PI511383 and PI560536) and 17 Pst-susceptible $\mathrm{F}_{2}$ plants, ten from popA and seven from popB (Supplementary Table 1), using the wheat 10k iSelect array ${ }^{29}$. The Pst responses of the tested plants were obtained in the field in 2011. For SNP data, we sequentially eliminated: (1) those with missing data or that were being heterozygous in the parents, (2) those that were being polymorphic between the two resistant parents or between the two susceptible lines, (3) those that were identical among the four parents, and (4) those with four or more missing data points amongst 17 Pst-susceptible $\mathrm{F}_{2}$ plants. We retained 3276 SNP loci for BSA analysis. Among Pst-susceptible $\mathrm{F}_{2}$ plants with a clear genotype, the frequency of a homozygous "B" genotype (= susceptible phenotype) was calculated and sorted in descending order for each SNP. The top 20 SNPs were prioritized for further analysis.

\section{Preliminary and fine mapping of the YrAS2388 gene. We targeted the} AT4D3406 region (Supplementary Fig. 1a) to develop PCR markers, which was facilitated by using the Ae. tauschii SNP map ${ }^{29}$, and the genome sequences of Ae. tauschii ${ }^{30}$, common wheat (IWGSC RefSeq v1.0 $0^{60}$ ), synthetic wheat ${ }^{35}$ and 20 fosmid clones of PI511383. Markers were primarily based on insertion-deletion polymorphisms (InDel), cleaved amplified polymorphic sequences (CAPS) and derived cleaved amplified polymorphic sequences $\left(\mathrm{dCAPS}^{61}\right)$. PCR primers, restriction enzymes and annealing temperatures are described in the Supplementary Table 2. All other oligos used in the current study are documented in the Supplementary Table 8 . PCR products were separated in either 6\% non-denaturing acrylamide or $2 \%$ agarose gels. The 4DS maps (Supplementary Fig. 1) were calculated using the maximum likelihood algorithm and the Kosambi function in JoinMap 4.0 (Kyazma B.V., Wageningen, Netherlands) and were assembled using MapChart v2.3 (www.wur.nl/en/show/Mapchart.htm).

Construction and screening of the fosmid genomic library. PI511383 leaf tissue was harvested from 4 -week-old plants and stored at $-80^{\circ} \mathrm{C}$. Megabase-size DNA was prepared by embedding nuclei in $0.5 \%$ low-melting agarose, followed by nuclear lysis in the presence of detergent and proteinase- $\mathrm{K}^{62}$. Sixty DNA plugs were transferred to individual 1.5 -ml tubes with $200-\mu \mathrm{l} \mathrm{TE}$ buffer. DNA in agarose was sheared by 22 freeze-thaw cycles with incubation in liquid nitrogen for $20 \mathrm{~s}$ and then a $45^{\circ} \mathrm{C}$ water bath for $3 \mathrm{~min}$. The sheared DNA in a 33.5-63.5-kb range was purified from a gel, repaired using the DNA End-Repair enzyme, ligated into the pCC1FOS vector, and packed into the phage particles as instructed by the CopyControl ${ }^{\text {mat }}$ Fosmid Library Production Kit (Epicentre Technologies Corp., Madison, WI, USA). Packaged fosmid clones were transformed into the EPI300-T1R competent cells, and the titer of the genomic library was calculated as indicated in the manual (Epicentre). On average, 1,000 or 2,000 clones per plate were obtained from a diluted solution after an $18 \mathrm{~h}$ to $24 \mathrm{~h}$ incubation at $37^{\circ} \mathrm{C}$. Colonies were recovered using a mix with $6 \mathrm{ml} \mathrm{LB}$ and $1.8 \mathrm{ml}$ glycerol, divided into three aliquots ( $2 \mathrm{ml}$ each, super colony pools), and stored at $-80{ }^{\circ} \mathrm{C}$

PCR screening was performed on each of 622 super colony pools, with $2-\mu \mathrm{l}$ bacterium stock as template. We screened for markers Xsdau 93 , Xsdau95 and $O 13$ (PCR primers P160/P161) (Supplementary Tables 2 and 8). PCR amplification was performed as follows: $95^{\circ} \mathrm{C}$ for $5 \mathrm{~min}, 32$ cycles with $95^{\circ} \mathrm{C}$ for $30 \mathrm{~s}, 58^{\circ} \mathrm{C}$ for $30 \mathrm{~s}$ and $72{ }^{\circ} \mathrm{C}$ for $50 \mathrm{~s}$, and a final extension at $72{ }^{\circ} \mathrm{C}$ for $10 \mathrm{~min}$. PCR products were separated on a $1 \%$ agarose gel and visualized by ethidium bromide staining. For positive super colony pools, 25 - $\mu$ l glycerol stock was inoculated into 5 -ml liquid LB supplemented with $12.5 \mu \mathrm{g}$ chloramphenicol ml $\mathrm{ml}^{-1}$ (LB-C), and cultured on a $250 \mathrm{rpm}$ shaker at $37^{\circ} \mathrm{C}$ for $4 \mathrm{~h}$. The culture was diluted in a 10 -fold series $\left(10^{-1}\right.$ to $10^{-5}$ ) using liquid $\mathrm{LB}$, and the serial dilutions $(300 \mu \mathrm{l}$ per level) were plated onto the LB-C agar. An ideal dilution yielded 4,000-5,000 clones per 15-cm-diameter plate, from which colonies were collected using the 384-pin replicator with four repeated contacts to collect more representative colonies. After the replicator was used to inoculate a 384-well plate with 50- $\mu$ liquid LB-C, the plate was incubated at $37^{\circ} \mathrm{C}$ overnight. Each well was screened by PCR. For positive wells, $20-\mu \mathrm{l}$ culture was enriched in 2-ml liquid LB-C, and grown in a $250 \mathrm{rpm}$ shaker at $37^{\circ} \mathrm{C}$ for $2 \mathrm{~h}$ The end culture was diluted 10 -fold (from $10^{-1}$ to $10^{-4}$ ) using liquid LB-C, and $100-\mu \mathrm{l}$ culture was plated onto LB-C agar. An ideal dilution yielded 50-200 clones per $9-\mathrm{cm}$-diameter plate, from which a positive clone would be revealed among 24 clones.

Mutagenesis and mutation screening. Synthetic hexaploid wheat SW3 and SynSAU-93 were treated with $0.8 \%$ EMS ( $78 \mathrm{mM}$ in water; Sigma-Aldrich Co., St. Louis, MO, USA). Briefly, lots of 400 seeds $\left(\mathrm{M}_{0}\right)$ were soaked in $100 \mathrm{ml}$ EMS solution, treated on a shaker at $150 \mathrm{rpm}$. at $25^{\circ} \mathrm{C}$ for $10 \mathrm{~h}$, and washed with running water at room temperatures for $4 \mathrm{~h} . \mathrm{M}_{1}$ plants of SW3 were grown in a greenhouse in Taian, China. $\mathrm{M}_{1}$ plants of Syn-SAU-93 were grown in the field in Chongzhou, China. To simplify the fieldwork, mutant seeds of SW3 and Syn-SAU93 were bulk planted at $M_{2}$ to $M_{4}$ generations in Chongzhou and Wenjiang, respectively, and screened for Pst resistance using mixed urediniospores of races CYR30, CYR31, CYR32, CYR33, Gui22-1, SY11-4, and HY46-8 in 2016-2018.

The Pst-susceptible mutants were screened for structural variations in the coding sequence of $R L K_{4 D S-1}, R L K_{4 D S-2}$, and $N L R_{4 D S-1}$. Plant DNA was prepared from the flag leaf using the Sarkosyl method ${ }^{17}$. Mutations of the candidate genes were identified using PCR-based DNA sequencing. For $R L K_{4 D S-1}$, we divided the 2570-bp fragment into two parts: (1) exons 1-3 between P162 and P163, and (2) exon 4 between P164 and P165. For $R L K_{4 D S-2}$, we examined a 1430-bp target region of the exon 3 between P167 and P168. For $N L R_{4 D S-1}$, we divided the 6072-bp fragment into five parts: (1) promoter and exons 1-3 between P169 and P170, (2) exon 4 between P171 and P172, (3) exon 5 between P173 and P174, (4) exon 5-6 between P175 and P176, and (5) the insertion region with $3^{\prime} \mathrm{UTR} 2$ between P177 and P178. PCR primers are described in the Supplementary Table 8. A standard PCR reaction was performed with Taq polymerase (Promega, Madison, WI, USA) The PCR products were sequenced by the Sangon Biotech Company (Chengdu, Sichuan, China).

Genetic transformation of wheat and barley. The fosmid PC1104 (= F2-1; Supplementary Table 4) from PI511383 is about $47.7 \mathrm{~kb}$ (GenBank MK288012). PC1104 contains genomic copies of $R L K_{4 D S-1}, R L K_{4 D S-2}$ and $N L R_{4 D S-1}$. Both intact and/or the restriction enzyme-cleaved PC1104 were used for wheat and barley transformation. For genetic transformations, we used four plasmids: (1) PC1104 for native expression of $R L K_{4 D S-1}, R L K_{4 D S-2}$, and $N L R_{4 D S-1}$, (2) BsrGI, NotI, XbaI, $K p n \mathrm{I}$, or $\mathrm{Xba \textrm {I }}+K p n \mathrm{I}$-cleaved PC1104 for native expression of $R L K_{4 D S-1}, R L K_{4 D S-2}$ and $N L R_{4 D S-1}$, (3) PC1101 for overexpression of the NLR $4 D S-1$ TV1 $\mathrm{CDNA}$, and (4) PC1102 for overexpression of the NLR ${ }_{4 D S-1} T_{V 2}$ CDNA (Supplementary Table 4). To overexpress the candidate genes, we cloned the cDNA copies of $N L R_{4 D S-1} T V 1$ cDNA with PCR primers P181/P182 and NLR 4 DS-1 TV2 CDNA with PCR primers P181/P183. We then assembled two plant expression constructs: PC1101 (Ubi:: $\left.N L R_{4 D S-1} T V 1-c D N A\right)$ and PC1102 (Ubi::NLR $4 D S-1$ TV2-cDNA) (Supplementary Table 4). The fosmid PC1104 has no plant selection marker, and thus required cotransformation with PC174, which has the bialaphos $(B A R)$ and hygromycin (HYG) selection markers both under the CaMV $35 S$ promoter (Supplementary Table 4). The other two plant expression constructs (PC1101 and PC1102) have both $B A R$ and $H Y G$ selection markers on their T-DNA, and were used for direct transformation.

Standard methods for biolistic bombardment and tissue culture of wheat were used $^{63}$. Using an intact fosmid PC1104, we bombarded 1,590 immature embryos of CB037 and generated 24 putative transgenic plants. Using the cleaved fosmid PC1104 (Supplementary Fig. 6), we bombarded 5,790 immature embryos of CB037 and 2,013 immature embryos of Bobwhite, and generated 197 and 20 putative 
transgenic plants, respectively. Using the bombardment protocol for wheat ${ }^{63}$, we also transferred the intact fosmid PC1104 into barley Golden Promise, however the tissue culture and regeneration procedures were specific for barley ${ }^{64}$. We bombarded 2,200 immature embryos of Golden Promise and generated 300 putative transgenic plants.

We also overexpressed the $N L R_{4 D S-1} \mathrm{cDNA}$ under the maize $U b i$ promoter in wheat and barley. For the $N L R_{4 D S-1} T V 1$ cDNA (in PC1101), we bombarded 2,200 wheat immature embryos (Bobwhite or CB037), obtained 40 putative $\mathrm{T}_{0}$ plants, and tested nine $N L R_{4 D S-1} T V 1$ expressing $\mathrm{T}_{1}$ families (seven of Bobwhite and two of CB037) for their response to PSTv-40. Using a standard Agrobacterium-mediated transformation ${ }^{64}$, we then infected 800 barley immature embryos (Golden Promise), obtained 15 putative transgenic $\mathrm{T}_{0}$ plants, and tested four $N L R_{4 D S-1} T V 1$ expressing $\mathrm{T}_{1}$ families against PSH-72. For $N L R_{4 D S-1} T V 2$ CDNA (PC1102), we bombarded 2,500 wheat immature embryos (Bobwhite), obtained 54 putative $\mathrm{T}_{0}$ plants, and tested ten $N L R_{4 D S-1} T V 2$ expressing $\mathrm{T}_{1}$ families for their response to PSTv-40. We also then infected 800 barley immature embryos (Golden Promise), obtained 28 putative transgenic $\mathrm{T}_{0}$ plants and tested $13 N_{N L R_{4 D S-1}} T_{V 2}$ expressing $\mathrm{T}_{1}$ families against PSH-72.

Transgene integration was confirmed by a positive amplification of $B A R$ with primers P184/P185, $R L K_{4 D S-1}$ with primers $\mathrm{P} 208 / \mathrm{P} 209, R L K_{4 D S-2}$ with primers P203/P204 and $N L R_{4 D S-1}$ with primers P213/P214 (or in the overexpression experiment with primers P186/P190). Transcription was assessed by RT-PCR with primers P208/P209 for $R L K_{4 D S-1}$, primers $\mathrm{P} 187 / \mathrm{P} 188$ for $R L K_{4 D S-2}$, and primers P189/P190 for NLR $R_{4 D S-1}$. ACTIN primers P191/P192 were used as an internal control for both wheat and barley. PCR primers are described in Supplementary Table 8.

Haplotype analysis. Haplotype analysis was performed to understand the association of haplotypes and responses to Pst and the evolution of the YrAS2388 region. Haplotype markers (HTM) were specifically designed for $R L K_{4 D S-1}$, $R L K_{4 D S-2}$ and $N L R_{4 D S-1}$. Their physical locations (in Supplementary Tables 3 and 5 , Supplementary Data 3) are counted from "A" in the start codon (ATG) in the genomic allele (GenBank accession number MK288012); for each marker, two periods separate the starting and ending nucleotides, and a minus sign indicates a backward count from " $\mathrm{A}$ " and a plus sign indicates a forward count from " $\mathrm{A}$ ". First, 159 Ae. tauschii accessions were genotyped in Sichuan, China using seven markers: HTM1a $\left(=R L K_{4 D S-1}\right)$, HTM2a $\left(=R L K_{4 D S-2}\right)$ and HTM3a to HTM3e $\left(=N L R_{4 D S-1}\right)$ (Supplementary Table 3, Supplementary Data 3). Second, 874 Triticeae lines were genotyped in Shandong, China using four markers: HTM1b $\left(=R L K_{4 D S-1}\right)$, HTM2b $\left(=R L K_{4 D S-2}\right)$ and HTM3f to HTM3g $\left(=N L R_{4 D S-1}\right)$. PCR primers are described in Supplementary Table 2. Markers used to genotype the Triticeae collection in Shandong were different from those used for genotyping the Ae. tauschii collection in Sichuan. Genotypes per gene per accession were not necessarily identical between the two tested collections. Thus, grouping of haplotypes should be considered separately for these two collections.

Gene expression analysis. RT-PCR was used to detect the expression of $R L K 1_{4 D S-1}$, $R L K 1_{4 D S-2}, N L R_{4 D S-1}$ and ACTIN (internal control). Plants were maintained at $25^{\circ}$ $\mathrm{C}$ during the day and $15{ }^{\circ} \mathrm{C}$ at night with a $16 \mathrm{~h}$ photoperiod. Total RNA was extracted using TRIzol (Life Technologies, Grand Island, NY, USA). First strand cDNA was synthesized using the M-MLV Reverse Transcriptase (Invitrogen, Carlsbad, CA, USA). RT-PCR was conducted on the $2^{\text {nd }}$-leaf of the juvenile (twoleaf stage) plants. Primers used were P193/P194 for $R L K_{4 D S-1}$, P195/P196 for $R L K_{4 D S-2}$, P197/P198 for NLR $4 D S-1$ and P191/P192 for ACTIN. Phusion HighFidelity DNA Polymerase (Thermo Scientific, Wilmington, DE, USA) was used to perform the PCR reaction with 30 cycles for $A C T I N, N L R_{4 D S-1}$ and $R L K_{4 D S-1}$ and 38 cycles for $R L K_{4 D S-2}$.

Quantitative real-time PCR (qRT-PCR) was used to measure four transcript variants of $N L R_{4 D S-1}$. QRT-PCR was conducted with SYBR Green reagents (Applied Biosystems, Foster City, CA, USA) on a StepOne Plus PCR System (Applied Biosystems). Specific PCR primers (Supplementary Table 8) were designed for four identified transcripts of the $N L R_{4 D S-1}$ gene. Wheat $A C T I N$ was used as an endogenous control 65 . Primers used were $\mathrm{P} 215 / \mathrm{P} 216$ for $N L R_{4 D S-1} T_{V 1}, \mathrm{P} 217 / \mathrm{P} 218$ for NLR ${ }_{4 D S-1} T_{T V 2}, \mathrm{P} 219 / \mathrm{P} 220$ for NLR $4 D S-1$ TV3, P221/P222 for NLR $4 D S-1$ TV4+ and P223/P224 for ACTIN. TV4+ also contains TV3, but TV3 only accounts for $2-5 \%$ of the total transcripts. The primer efficiencies were between $90 \%$ and $105 \%$. Transcript levels were expressed as linearized fold-ACTIN levels calculated by the

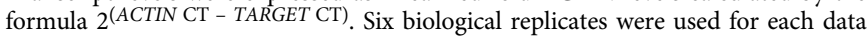
point. Data were analyzed using the SAS program (v9.4).

Sequence analysis. Fosmid clones were extracted using QIAGEN Large Construct Kits (QIAGEN, Germantown, MD, USA). Library preparation, highthroughput sequencing and quality control were performed by the Berry Genomics Company (Beijing, China). In brief, DNA was fragmented, endrepaired, ligated to Illumina adaptors, and separated on a $2 \%$ agarose gel to select fragments about $400-500 \mathrm{bp}^{66}$. Adaptor specific primers were used to amplify the ligation products. The final library was evaluated by qRT-PCR. PE reads $(150 \mathrm{bp})$ were obtained using the Illumina HiSeq2500. Sequence reads of the vector pCC1FOS and the bacterial genome were masked by the crossmatch tool in the Phrap package ${ }^{67}$. A de novo assembly of each fosmid was done using either SPAdes 3.12 [http://cab.spbu.ru/software/spades/] or ABySS 2.0.2 [www. bcgsc.ca/platform/bioinfo/software/abyss/releases/2.0.2]. Orientation and order of small contigs was inferred using the reference sequences of W7984 ${ }^{35}$ and AL8/ $78^{30}$. Sequence gaps were filled by PCR clones and Sanger sequencing. Candidate genes were identified by searching Ensemble Plants [http://plants.ensembl.org/ index.html] and the NCBI databases [http://www.ncbi.nlm.nih.gov/]. The secondary and three-dimensional structures of the $\mathrm{NLR}_{4 \mathrm{DS}-1}$ protein were predicted using PSIPRED [http://bioinf.cs.ucl.ac.uk/psipred/] and Phyre2 [http://www.sbg. bio.ic.ac.uk/phyre2/], respectively.

Reporting summary. Further information on research design is available in the Nature Research Reporting Summary linked to this article.

\section{Data availability}

Data supporting the findings of this work are available within the paper and its Supplementary Information files. A reporting summary for this Article is available as a Supplementary Information file. GenBank accessions include MK288012 for the fosmid PC1104 (= F2-1), MK288013 for the YrAS2388R contig, MK736661 for the YrAS2388R gene in PI511383, MK736662 for the YrAS2388R gene in Clae9, MK736663 for the YrAS2388R gene in PI511384 (= AS2388), MK736664 for the YrAS2388S gene in PI560536, MK736665 for the YrAS2388S gene in PI486274, and MK736666 for the YrAS2388S gene in AS87. Source data underlying Figs. 1a and 2, as well as Supplementary Figs. 3-5, 7b, and 11 are provided as a Source Data file. All datasets generated and analyzed during the current study are available from the corresponding author upon request.

Received: 15 January 2019 Accepted: 5 August 2019

Published online: 06 September 2019

\section{References}

1. WAP. World agricultural production. In: Circular Series, WAP 03-19. (United States Department of Agriculture-Foreign Agricultural Service, 2019). https://apps.fas.usda.gov/psdonline/circulars/production.pdf.

2. Shewry, P. R. \& Hey, S. J. The contribution of wheat to human diet and health. Food Energy Secur. 4, 178-202 (2015).

3. United-Nations. World population prospects: The 2015 revision, Key findings and advance tables. (Department of Economic and Social Affairs PD, United Nations, 2015). https://www.un.org/en/development/desa/publications/worldpopulation-prospects-2015-revision.html

4. Chen, W., Wellings, C., Chen, X., Kang, Z. \& Liu, T. Wheat stripe (yellow) rust caused by Puccinia striiformis f. sp. tritici. Mol. Plant Pathol. 15, 433-446 (2014).

5. Solh M., Nazari K., Tadesse W., Wellings C. R. The growing threat of stripe rust worldwide. In: Borlaug Global Rust Initiative, 2012 Technical Workshop, Beijing, China (ed. McIntosh R. A.) (Borlaug Global Rust Initiative, 2012). https://www.globalrust.org/sites/default/files/posters/solh_2012.pdf.

6. Ali, S. et al. Yellow rust epidemics worldwide were caused by pathogen races from divergent genetic lineages. Front. Plant Sci. 8, 1057 (2017).

7. Chen, X. M. Epidemiology and control of stripe rust (Puccinia striiformis f. sp. tritici) on wheat. Canadian. J. Plant Pathol. 27, 314-337 (2005).

8. de Vallavieille-Pope, C. et al. Virulence dynamics and regional structuring of Puccinia striiformis f. sp. tritici in France between 1984 and 2009. Plant Dis. 96, 131-140 (2011).

9. Bayles, R. A., Flath, K., Hovmøller, M. S. \& Vallavieille-Pope, Cd Breakdown of the Yr17 resistance to yellow rust of wheat in northern Europe. Agronomie 20, 805-811 (2000).

10. Chen, X. High-temperature adult-plant resistance, key for sustainable control of stripe rust. Am. J. Plant Sci. 4, 608-627 (2013).

11. Gessese, M., Bariana, H., Wong, D., Hayden, M. \& Bansal, U. Molecular mapping of stripe rust resistance gene $Y r 81$ in a common wheat landrace Aus27430. Plant Dis. 103, 1166-1171 (2019).

12. Marchal, C. et al. BED-domain-containing immune receptors confer diverse resistance spectra to yellow rust. Nat. Plants 4, 662-668 (2018).

13. Klymiuk, V. et al. Cloning of the wheat Yr15 resistance gene sheds light on the plant tandem kinase-pseudokinase family. Nature. Communications 9, 3735 (2018).

14. Fu, D. et al. A kinase-START gene confers temperature-dependent resistance to wheat stripe rust. Science 323, 1357-1360 (2009).

15. Krattinger, S. G. et al. A putative $\mathrm{ABC}$ transporter confers durable resistance to multiple fungal pathogens in wheat. Science 323, 1360-1363 (2009).

16. Moore, J. W. et al. A recently evolved hexose transporter variant confers resistance to multiple pathogens in wheat. Nat. Genet. 47, 1494-1498 (2015). 
17. Yuan, C. et al. Distribution, frequency and variation of stripe rust resistance loci $Y r 10, L r 34 / Y r 18$ and Yr36 in Chinese wheat cultivars. J. Genet. Genom. 39, 587-592 (2012).

18. Wan, A. \& Chen, X. Virulence characterization of Puccinia striiformis $\mathrm{f}$. sp. tritici using a new set of $\mathrm{Yr}$ single-gene line differentials in the United States in 2010. Plant Dis. 98, 1534-1542 (2014).

19. Chen, X., Penman, L., Wan, A. \& Cheng, P. Virulence races of Puccinia striiformis f. sp. tritici in 2006 and 2007 and development of wheat stripe rust and distributions, dynamics, and evolutionary relationships of races from 2000 to 2007 in the United States. Canadian. J. Plant Pathol. 32, 315-333 (2010).

20. Wang, J. et al. Aegilops tauschii single nucleotide polymorphisms shed light on the origins of wheat D-genome genetic diversity and pinpoint the geographic origin of hexaploid wheat. New Phytol. 198, 925-937 (2013).

21. McFadden, E. S. \& Sears, E. R. The origin of Triticum spelta and its freethreshing hexaploid relatives. J. Hered. 37, 81-89 (1946).

22. Liu, M. et al. Stripe rust resistance in Aegilops tauschii germplasm. Crop Sci. 53, 2014-2020 (2013).

23. Huang, L. et al. Molecular tagging of a stripe rust resistance gene in Aegilops tauschii. Euphytica 179, 313-318 (2011).

24. Singh, R. P., Nelson, J. C. \& Sorrells, M. E. Mapping Yr28 and other genes for resistance to stripe rust in wheat. Crop Sci. 40, 1148-1155 (2000).

25. Zhang, R. et al. Two main stripe rust resistance genes identified in syntheticderived wheat line Soru\#1. Phytopathology 109, 120-126 (2018).

26. Zhang, H.-Q., Jia, J.-Z., Yang, H. \& Zhang, B.-S. SSR mapping of stripe rust resistance gene from Ae. tauschii. Hered. (Beijing) 30, 491-494 (2008).

27. Ogbonnaya F. C., et al. Synthetic hexaploids: Harnessing species of the primary gene pool for wheat improvement. In: Plant Breeding Reviews (ed. Janick J.) John Wiley \& Sons, Inc (2013). https://onlinelibrary.wiley.com/ doi/abs/10.1002/9781118497869.ch2.

28. GHJ, Kema, Lange, W. \& CHV, Silfhout Differential suppression of stripe rust resistance in synthetic wheat hexaploids derived from Triticum turgidum subsp. dicoccoides and Aegilops squarrosa. Phytopathology 85, 425-429 (1995).

29. Luo, M.-C. et al. A 4-gigabase physical map unlocks the structure and evolution of the complex genome of Aegilops tauschii, the wheat D-genome progenitor. Proc. Natl Acad. Sci. 110, 7940-7945 (2013).

30. Luo, M.-C. et al. Genome sequence of the progenitor of the wheat D genome Aegilops tauschii. Nature 551, 498-502 (2017).

31. Afzal, A. J., Wood, A. J. \& Lightfoot, D. A. Plant receptor-like serine threonine kinases: Roles in signaling and plant defense. Mol. Plant-Microbe Interact. 21, 507-517 (2008).

32. Friesen, T. L., Xu, S. S. \& Harris, M. O. Stem rust, tan spot, stagonospora nodorum blotch, and hessian fly resistance in Langdon durum-Aegilops tauschii synthetic hexaploid wheat lines. Crop Sci. 48, 1062-1070 (2008).

33. Zhang, L.-Q. et al. Frequent occurrence of unreduced gametes in Triticum turgidum-Aegilops tauschii hybrids. Euphytica 172, 285-294 (2010).

34. Peña, P. A. et al. Molecular and phenotypic characterization of transgenic wheat and sorghum events expressing the barley alanine aminotransferase. Planta 246, 1097-1107 (2017).

35. Chapman, J. A. et al. A whole-genome shotgun approach for assembling and anchoring the hexaploid bread wheat genome. Genome Biol. 16, 26 (2015).

36. Keidar-Friedman, D., Bariah, I. \& Kashkush, K. Genome-wide analyses of miniature inverted-repeat transposable elements reveals new insights into the evolution of the Triticum-Aegilops group. PLOS ONE 13, e0204972 (2018).

37. Brar, G. S., Dhariwal, R. \& Randhawa, H. S. Resistance evaluation of differentials and commercial wheat cultivars to stripe rust (Puccinia striiformis) infection in hot spot regions of Canada. Eur. J. Plant Pathol. 152, 493-502 (2018).

38. Knaggs, P., Ambrose, M. J., Reader, S. M. \& Miller, T. E. Morphological characterisation and evaluation of the subdivision of Aegilops tauschii Coss. Wheat Inf. Serv. 91, 15-19 (2000).

39. Yildirim, A., Jones, S. S., Murray, T. D., Cox, T. S. \& Line, R. F. Resistance to stripe rust and eyespot diseases of wheat in Triticum tauschii. Plant Dis. 79, 1230-1236 (1995).

40. Wang M., Chen X. Stripe rust resistance. In: Stripe Rust (eds Chen X., Kang Z.) (Springer, Netherlands, 2017)

41. Klindworth, D. L., Hareland, G. A., Elias, E. M. \& Xu, S. S. Attempted compensation for linkage drag affecting agronomic characteristics of durum wheat 1AS/1DL translocation lines. Crop Sci. 53, 422-429 (2013).

42. Riley, R., Chapman, V. \& Johnson, R. O. Y. Introduction of yellow rust resistance of Aegilops comosa into wheat by genetically induced homoeologous recombination. Nature 217, 383-384 (1968).

43. Oak, M. D. \& Tamhankar, S. A. 1BL/1RS translocation in durum wheat and its effect on end use quality traits. J. Plant Biochem. Biotechnol. 26, 91-96 (2017).

44. Qie, Y. et al. Development, validation, and re-selection of wheat lines with pyramided genes Yr64 and Yr15 linked on the short arm of chromosome 1B for resistance to stripe rust. Plant Dis. 103, 51-58 (2018).
45. Kuersten, S. \& Goodwin, E. B. The power of the 3' UTR: translational control and development. Nat. Rev. Genet. 4, 626-637 (2003).

46. Lianoglou, S., Garg, V., Yang, J. L., Leslie, C. S. \& Mayr, C. Ubiquitously transcribed genes use alternative polyadenylation to achieve tissue-specific expression. Genes Dev. 27, 2380-2396 (2013).

47. Kornblihtt, A. R. et al. Alternative splicing: a pivotal step between eukaryotic transcription and translation. Nat. Rev. Mol. Cell Biol. 14, 153-165 (2013).

48. Pan, Q., Shai, O., Lee, L. J., Frey, B. J. \& Blencowe, B. J. Deep surveying of alternative splicing complexity in the human transcriptome by highthroughput sequencing. Nat. Genet. 40, 1413-1415 (2008).

49. Howard, B. E. et al. High-throughput RNA sequencing of Pseudomonasinfected Arabidopsis reveals hidden transcriptome complexity and novel splice variants. PLOS ONE 8, e74183 (2013).

50. Zhang, X.-C. \& Gassmann, W. Alternative splicing and mRNA levels of the disease resistance gene RPS4 are induced during defense responses. Plant Physiol. 145, 1577-1587 (2007).

51. Yan, L. \& Thomas, E. Transcript-level expression control of plant NLR genes. Mol. Plant Pathol. 19, 1267-1281 (2018).

52. Hao, W., Collier, S. M., Moffett, P. \& Chai, J. Structural basis for the interaction between the potato virus $\mathrm{X}$ resistance protein $(\mathrm{Rx})$ and its cofactor Ran GTPase-activating protein 2 (RanGAP2). J. Biol. Chem. 288, 35868-35876 (2013).

53. Maekawa, T., Kufer, T. A. \& Schulze-Lefert, P. NLR functions in plant and animal immune systems: So far and yet so close. Nat. Immunol. 12, 817-826 (2011).

54. Deng, Y. et al. Epigenetic regulation of antagonistic receptors confers rice blast resistance with yield balance. Science 355, 962-965 (2017)

55. Williams, N. D., Miller, J. D. \& Klindworth, D. L. Induced mutations of a genetic suppressor of resistance to wheat stem rust. Crop Sci. 32, 612-616 (1992).

56. Daniel, S. et al. Suppression among alleles encoding nucleotidebinding-leucine-rich repeat resistance proteins interferes with resistance in $F_{1}$ hybrid and allele-pyramided wheat plants. Plant J. 79, 893-903 (2014).

57. Hurni, S. et al. The powdery mildew resistance gene $P m 8$ derived from rye is suppressed by its wheat ortholog. Pm3. Plant J. 79, 904-913 (2014).

58. Baker, R. J. \& Dyck, P. L. Combining ability for yield of synthetic hexaploid wheats. Can. J. Plant Sci. 54, 235-239 (1974).

59. Line, R. F. \& Qayoum, A. Virulence, aggressiveness, evolution, and distribution of races of Puccinia striiformis (the cause of stripe rust of wheat) in North America, 1968-87. US Dep. Agric Tech. Bull. 1788, 44 (1992).

60. IWGSC. Shifting the limits in wheat research and breeding using a fully annotated reference genome. Science 361, eaar7191 (2018).

61. Neff, M. M., Neff, J. D., Chory, J. \& Pepper, A. E. dCAPS, a simple technique for the genetic analysis of single nucleotide polymorphisms: experimental applications in Arabidopsis thaliana genetics. Plant J. 14, 387-392 (1998)

62. Luo M., Wing R. A. An improved method for plant BAC library construction. In: Methods in Molecular Biology (ed. Grotewold E.) (2003). https://link. springer.com/protocol/10.1385\%2F1-59259-413-1\%3A3.

63. Lv, B. et al. Characterization of FLOWERING LOCUS T1 (FT1) gene in Brachypodium and wheat. PLoS ONE 9, e94171 (2014).

64. Harwood W. A., et al. Barley transformation using Agrobacterium-mediated techniques. In: Transgenic Wheat, Barley And Oats: Production And Characterization Protocols (eds Jones D. H., Shewry R. P.) (Humana Press, 2009). https://link.springer.com/protocol/10.1007/978-1-59745-379-0_9.

65. Fu, D., Dunbar, M. \& Dubcovsky, J. Wheat VIN3-like PHD finger genes are up-regulated by vernalization. Mol. Genet. Genom. 277, 301-313 (2007).

66. Ni, F. et al. Wheat $M s 2$ encodes for an orphan protein that confers male sterility in grass species. Nature. Communications 8, 15121 (2017).

67. Ewing, B., Hillier, L., Wendl, M. C. \& Green, P. Base-calling of automated sequencer traces using Phred. I. Accuracy assessment. Genome Res. 8, 175-185 (1998).

68. Christensen, A. H., Sharrock, R. A. \& Quail, P. H. Maize polyubiquitin genes: Structure, thermal perturbation of expression and transcript splicing, and promoter activity following transfer to protoplasts by electroporation. Plant Mol. Biol. 18, 675-689 (1992)

\section{Acknowledgements}

We thank Miss Y. Liu for her assistance with Pst spores and Mengmeng Lin for her assistance in taking photos of infected plants. The data reported in this paper are tabulated in the Supplementary Materials and/or archived as GenBank accessions as indicated in the main text. This work is supported by the China Research and Development Initiative on Genetically Modified Plants (2016ZX08009003-001-006), the National Key Research and Development Program of China (2016YFD0102000), and Hatch project IDA01587 from the USDA National Institute of Food and Agriculture. The contents are solely the responsibility of the authors and do not necessarily represent the official views of the USDA or NIFA. 


\section{Author contributions}

D.F. conceived the project; D.L., J.Wa., J.Wu, L.E., M-C.L., X.C. and Y.Z. contributed ideas and resources, B.L., C.K., C.Z., F.C., F.N., G.G., H.Z., J.Q., L.H., L.Z., M.H., M.Li, M.Liu, M.W. and Q.H. performed the experiments; C.Z., D.F. and L.H. analyzed the data; C.Z., D.F. and L.E. wrote the paper; and all authors discussed the results and the paper.

\section{Additional information}

Supplementary Information accompanies this paper at https://doi.org/10.1038/s41467019-11872-9.

Competing interests: C.Z., D.F. D.L., J.Wu, F.N., G.G., H.Z., L.Z. and L.H declare the competing interest in the use of the $N L R_{4 D S-1}$ gene (China patent filing No.

201811424853.2). The remaining authors declare no competing interests.

Reprints and permission information is available online at http://npg.nature.com/ reprintsandpermissions/

Peer review information: Nature Communications thanks Kostya Kanyuka, Roger Wise and the other, anonymous, reviewer(s) for their contribution to the peer review of this work. Peer reviewer reports are available.
Publisher's note: Springer Nature remains neutral with regard to jurisdictional claims in published maps and institutional affiliations.

(c) (i) Open Access This article is licensed under a Creative Commons Attribution 4.0 International License, which permits use, sharing, adaptation, distribution and reproduction in any medium or format, as long as you give appropriate credit to the original author(s) and the source, provide a link to the Creative Commons license, and indicate if changes were made. The images or other third party material in this article are included in the article's Creative Commons license, unless indicated otherwise in a credit line to the material. If material is not included in the article's Creative Commons license and your intended use is not permitted by statutory regulation or exceeds the permitted use, you will need to obtain permission directly from the copyright holder. To view a copy of this license, visit http://creativecommons.org/ licenses/by/4.0/.

(C) The Author(s) 2019 